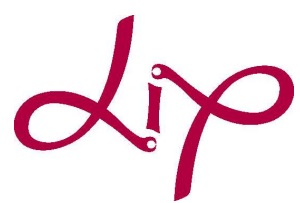

Laboratoire de l'Informatique du Parallélisme

Optimizing the reliability of pipelined applications under throughput constraints

Anne Benoit,

Hinde Lilia Bouziane,

February 2010

Yves Robert

Research Report No 2010-06

École Normale Supérieure de Lyon

46 Allée d'Italie, 69364 Lyon Cedex 07, France

Téléphone : $+33(0) 4.72 .72 .80 .37$

Télécopieur : +33(0)4.72.72.80.80

Adresse électronique : lip@ens-lyon.fr
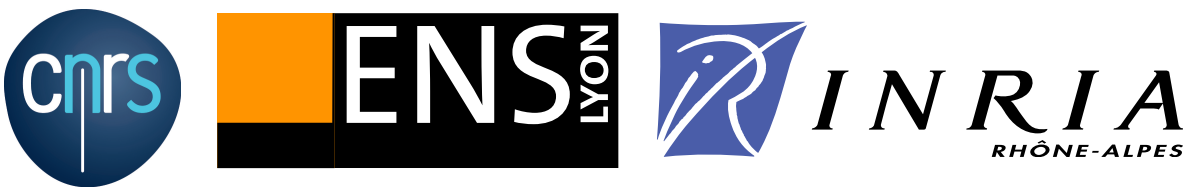


\title{
Optimizing the reliability of pipelined applications under throughput constraints
}

\author{
Anne Benoit, Hinde Lilia Bouziane, Yves Robert
}

February 2010

\begin{abstract}
Mapping a pipelined application onto a distributed and parallel platform is a challenging problem. The problem becomes even more difficult when multiple optimization criteria are involved, and when the target resources are heterogeneous (processors and communication links) and subject to failures. This report investigates the problem of mapping pipelined applications, consisting of a linear chain of stages executed in a pipeline way, onto such platforms. The objective is to optimize the reliability under a performance constraint, i.e., while guaranteeing a threshold throughput. In order to increase reliability, we replicate the execution of stages on multiple processors. We present complexity results, proving that this bi-criteria optimization problem is NP-hard. We then propose some heuristics, and present extensive experiments evaluating their performance.
\end{abstract}

Keywords: pipelined applications, interval mapping, throughput, reliability, heterogeneous platforms, bi-criteria optimization, complexity, heuristics.

\section{Résumé}

Le problème du placement d'applications sur des infrastructures matérielles distribuées et/ou parallèles représente un grand défi. Ce problème est encore plus difficile lorsque plusieurs critères à optimiser sont à prendre en compte et lorsque ces plates-formes sont hétérogènes (processeurs et liens de communication). Dans ce rapport, nous étudions le problème du placement d'applications pipelinées, composées d'une suite linéaire d'étages exécutés dans un mode pipeline, sur ce type de plates-formes. Notre objectif est d'optimiser la fiabilité sous contrainte de performance, c.-à-d, en assurant un débit minimum à l'exécution. Pour rendre l'application plus fiable, nous utilisons un mécanisme de réplication qui consiste à exécuter un étage de l'application sur plus d'un processeur. Nous présentons les résultats de complexité qui montrent que le présent problème d'optimisation bi-critère est NP-difficile. Nous proposons ensuite un ensemble d'heuristiques et présentons les résultats expérimentaux qui évaluent leurs performances.

Mots-clés: applications pipelinées, placement, débit, fiabilité, plates-formes hétérogènes, optimisation bi-critère, complexité, heuristiques. 


\section{Contents}

1 Introduction 2

2 The mapping problem through an example $\quad 3$

3 Framework 6

3.1 Applicative framework . . . . . . . . . . . . . . . . 6

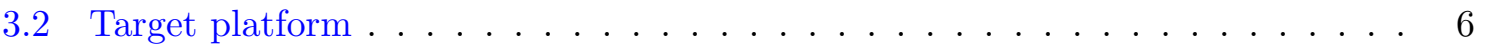

3.3 Communication model . . . . . . . . . . . . . . . . . 8

3.4 Mapping problem . . . . . . . . . . . . . . . . . . 8

4 Complexity results $\quad 10$

5 Mixed integer linear program formulation $\quad 12$

6 Heuristics $\quad 16$

6.1 Class 1: Heuristics partitioning then mapping . . . . . . . . . . . . . . 16

6.1 .1 Partitioning phase . . . . . . . . . . . . . . 16

6.1.2 Heuristics for mapping pre-defined intervals . . . . . . . . . . . . . 18

6.1.3 Partitioning-then-mapping heuristics . . . . . . . . . . . . . 20

6.2 Class 2: Heuristics with progressive creation of intervals . . . . . . . . . 21

7 Experiments $\quad 26$

7.1 Absolute performance of heuristics . . . . . . . . . . . . . . 26

7.2 Comparisons between heuristics . . . . . . . . . . . . . . 28

7.3 Summary . . . . . . . . . . . . . . . . . . . . . 29

8 Conclusion $\quad 35$ 


\section{Introduction}

Mapping applications onto distributed and parallel platforms is a difficult problem. The problem becomes even more difficult if we consider state-of-the-art heterogeneous platforms, like clusters or grids. These platforms are typically composed of processors with different speeds and interconnected through networks with different link capacities. In addition, they are subject to failures, which implies adding replication mechanisms to provide a more reliable application execution, and which in turn introduces another level of difficulty to the mapping problem.

A large number of programming models are proposed to design and develop distributed and parallel applications. These models offer means to deal with the complexity of such applications as well as with the complexity of the execution platforms, while attempting to ensure efficient execution and resource usage. In particular, algorithmic skeletons have been introduced to support typical parallel patterns [6, 10]. A skeleton follows a precise structure and execution behavior, like pipelined or farmed computations. It thus provides information that can help the realization of an efficient mapping. In this report, we focus on the widely used pipeline skeleton. A pipeline is a linear chain structure in which a stream of data enters a stage and progresses from stage to stage until the final result is computed. Each stage reads an input data produced by the previous stage, processes the data and outputs a result to the next stage. Finally, the pipeline operates in a synchronous mode: after an initialization delay, a new data in the stream is processed every period.

The mapping problem for a pipelined application can informally be stated as to choose which processor is assigned which stage. This choice may be done according to one or multiple optimization criteria. We focus on two key metrics: period (inverse of throughput) and reliability. The period of a mapping is defined as the longest cycle time of a processor. Under a bounded multiport platform model with overlap [8], i.e., in which a processor can simultaneously receive, compute and send data, the cycle time is the maximum among the times spent to perform these operations for all processed data. The reliability of an application is the probability that all computations will be successfully performed. The reliability is increased by replicating each stage on a set of processors. Thus, the application fails to be executed only if processors involved in the execution of a same stage all fail during the whole execution.

This report is a follow-on of a thread of papers aiming at period and/or reliability optimization. Computing a mapping minimizing the period has been studied in $[12,13]$ onto homogeneous platforms and later in [3] onto heterogeneous platforms. A first attempt to solve the period/reliability problem can be found in [2]. The work in [2] addresses a similar but different problem, that of replicating both for performance (assigning different data sets to different processors, to decrease the period) and for reliability (assigning the same data sets to different processors, to increase the reliability), at the price of a simplified model without any communication cost. In this report, we take communication costs into account, at the price on concentrating on the sole replication for reliability. Indeed, the impact of communications turns out to have dramatic consequences on the difficulty of the mapping problem. This report is the first attempt to deal with the induced combinatorial complexity of deciding for message originators and orchestrating communications in the period/reliability problem.

We follow an interval-mapping approach, where a processor is assigned a single interval of consecutive stages. We consider platforms with different-speed processors having either identical failure probabilities (failure homogeneous platforms) or different failure probabilities 
(fully heterogeneous platforms). The objective is to maximize the reliability under a period constraint onto such platforms.

The rest of the report is organized as follows. Section 2 illustrates the target optimization problem through an example, and further motivates this work. Section 3 formally details the mapping problem, and Section 4 establishes the complexity results. Next in Section 5, we introduce a linear program to solve the mapping problem onto failure homogeneous platforms. We also introduce several polynomial-time heuristics in Section 6 to propose practical solutions for the more general case of fully heterogeneous platforms. Section 7 is devoted to experimental results; we evaluate the absolute performance of the heuristics with respect to the linear program on small problem instances, before comparing their relative performance on larger instances (whose solution is inaccessible to the linear program). Finally, Section 8 provides some concluding remarks and directions for future work.

\section{The mapping problem through an example}

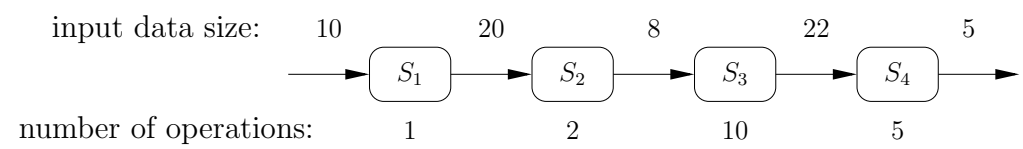

Figure 1: Example of a pipelined application with 4 stages $\left(S_{1} . . S_{4}\right)$.

This section aims at outlining the difficulty of mapping a pipelined application on a given execution platform. We consider the application example shown in Figure 1. For each stage of the pipeline, we specify the number of computations, and the size of input/output data.

Figure 2 shows the operations performed for the first six data sets $d_{1} . . d_{6}$ of the (possibly infinite) input stream. The mapping is assumed to be known: $S_{1}$ and $S_{2}$ are both assigned to same processors, while $S_{3}$ and $S_{4}$ are assigned to distinct processors. The notation $S_{i} \rightarrow$ $S_{i+1}$ corresponds to a remote communication from the processor assigned to $S_{i}$ to the one assigned to $S_{i+1}$. For a given data set, the stages are executed in a sequential way, while they are executed in pipelined fashion for different data sets. As soon as the first result res 1 is produced, the pipeline reaches a steady state and periodically produces a result. In the example, provided the threshold 5.5 for the the period of the mapping, i.e., the inverse of the throughput, is 5.5: a new data set enters in the pipeline every 5.5 time units.

The execution platform is shown in Figure 3. In this platform, processors are heterogeneous (different speeds and network card capacities) and are interconnected through homogeneous network links (identical bandwidth $b=5$ data units per time unit). Such a platform may well represent a heterogeneous cluster. In addition, we assume that processors are subject to unrecoverable failures, where each processor has a probability $f$ to fail during the whole application execution.

To map the application onto the platform, we need to define some rules. First, we assume that a processor can be assigned at most one set of consecutive stages. Such a set is named an interval. This rule is reasonable, as it allows to better exploit processor capabilities, and it may avoid costly communications. Second, we must handle failures to provide a failuretolerant execution scheme. For that, we adopt the well known replication principle, which consists in performing redundant executions of some, or all, application intervals on different 


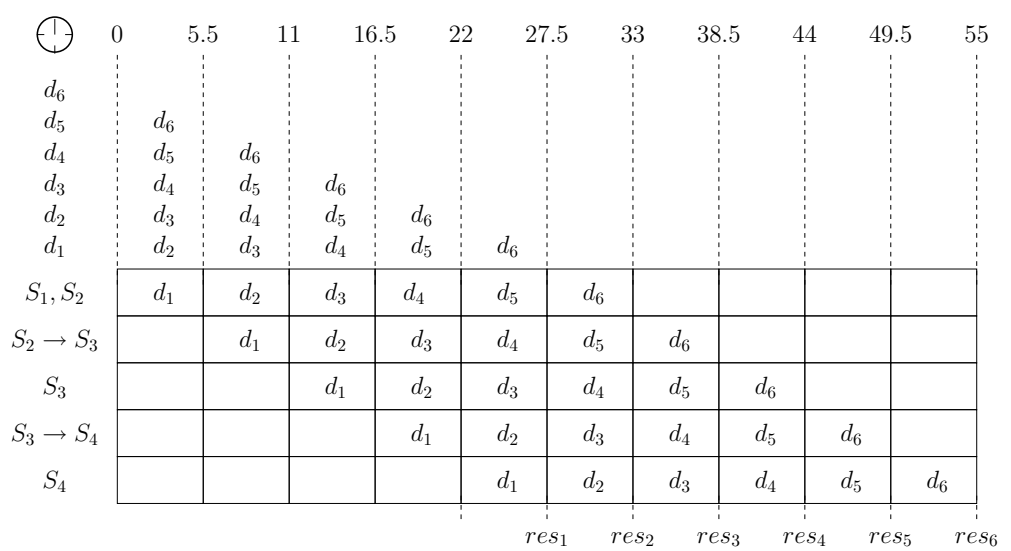

Figure 2: Periodic execution of the application shown in Figure $1 ; d_{1}, \ldots, d_{6}$ are the first six data sets input to the pipeline, and $r e s_{1}, \ldots$, res $_{6}$ are the corresponding results.

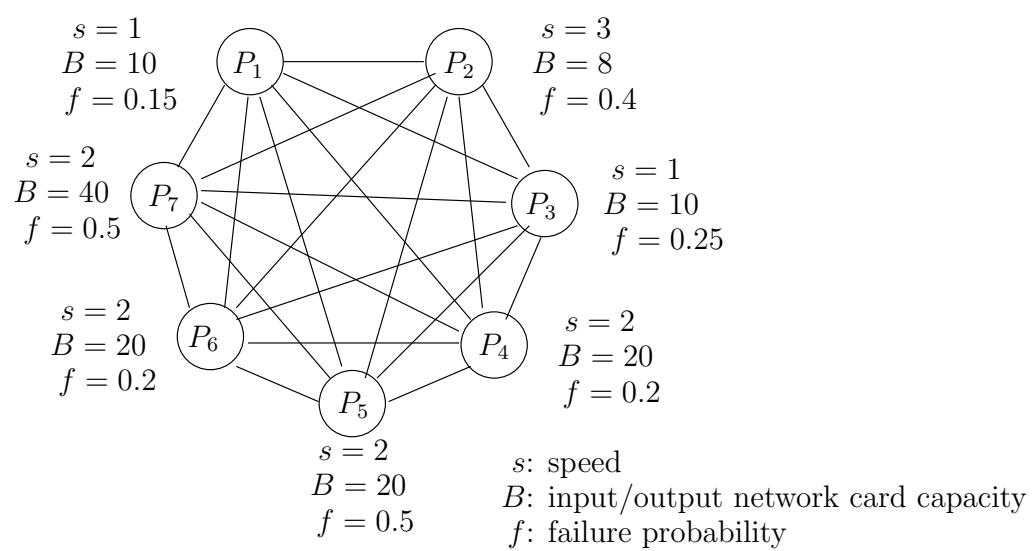

Figure 3: Target execution platform. The links between each processor pair have a bandwidth $b=5$.

processors. Therefore, a result will be produced (the one of the last stage in the interval) even if a processor fails. In order to avoid producing redundant results for the interval if two or more processors do not fail, we enforce a consensus protocol. This protocol is applied after the process of each data set for a given stage interval. The protocol elects one surviving processor as the sender of the output result of the interval to all processors executing the next interval. This election amounts to choosing the surviving processor allowing for the fastest output communications. We point out that communication links are assumed to be reliable: once a communication is initiated by a processor, it is achieved successfully, and no message is lost.

Considering these rules, mapping stages $S_{1}, \ldots, S_{4}$ on $P_{1}, \ldots, P_{7}$ raises the following questions: how to partition the stages into intervals and how to partition the processors over the intervals? The objective function consists in maximizing the reliability of the application, given a threshold on the period that should not be exceeded. It is a bi-criteria optimization problem: the goal is to find a mapping which maximizes the reliability with a constraint on the period. 
Figure 4 shows an optimal mapping for the example, when the threshold period set to $P_{\max }=5.5$. In this mapping, stages are partitioned into 3 intervals $\left[S_{1}, S_{2}\right],\left[S_{3}, S_{3}\right]$ and $\left[S_{4}, S_{4}\right]$. The period of the mapping is defined by the maximum cycle time of all processors $P_{1}, . ., P_{7}$. This cycle time is deduced from the periodic behavior illustrated by Figure 2, which assumes an overlap of communications and computations. For instance, the cycle time of processor $P_{1}$ is computed as follows:

$$
C T_{1}=\max \left(\max \left(\frac{10}{10}, \frac{10}{5}\right), \frac{1+2}{1}, \max \left(\frac{8 * 3}{10}, \frac{8}{5}\right)\right)=3
$$

The first term corresponds to input data (size 10, input network card 10, link bandwidth 5), the second to computations (sum of stage weights $1+2$ divided by speed 1 ), and the third to output data (size 8, sent 3 times, output network card 10, link bandwidth 5). Similarly, the cycle time of other processors are $C T_{2}=5.5, C T_{3}=5, C T_{4}=5, C T_{5}=5, C T_{6}=4.4$ and $C T_{7}=2$. Note that $P_{2}$ is the critical resource with the largest cycle time. This cycle time determines the period reachable in the case where processors $P_{4}$ and $P_{5}$ fail (Figure 5).

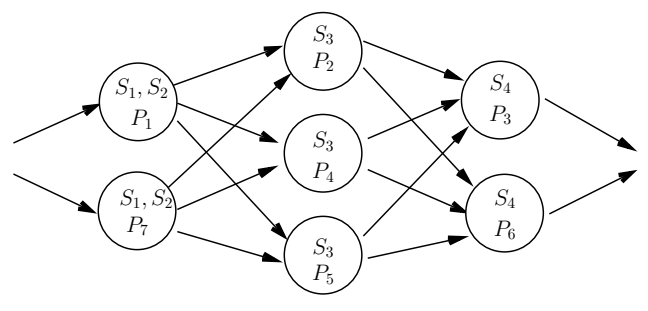

Figure 4: A mapping of the pipelined application of Figure 1 on the platform of Figure 3.

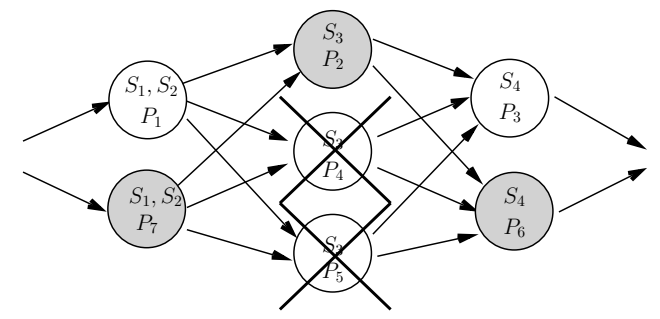

Figure 5: A failure configuration reaching the worst case period. $P_{4}$ and $P_{5}$ failed. The colored processors are those elected for sending the results.

Finally, the failure probability is computed as 1 minus the probability that the execution is successful, which happens if and only if all intervals are successful (hence a product in the formula); next a given interval fails if and only if all its assigned processors fail. In the mapping example we derive that

$$
\mathcal{F}=1-(1-0.15 * 0.5) *(1-0.4 * 0.2 * 0.5) *(1-0.25 * 0.2)=0.1564
$$

This latter probability turns out to be the minimum that can be obtained by all possible mapping solutions of the application example onto the current platform. To prove this, we have enumerated all possible mapping solutions. However, the number of these solutions is exponential. While it may be possible to evaluate all of them for small instances, this is not conceivable for real-life problems. To the best of our knowledge, there is no solution in the literature for this challenging bi-criteria reliability and period optimization problem, on a fully heterogeneous platform with communication costs. The following section presents more formally the applicative framework as well as the mapping problem. 


\section{Framework}

\subsection{Applicative framework}

This work focuses on pipelined applications. A pipeline is composed of $n$ ordered stages $S_{i}$, $1 \leq i \leq n$. These stages continuously operate on a stream of data. When input data are fed into the pipeline, they are processed from stage to stage, until they exit the last stage $S_{n}$. In other words, each stage $S_{i}$ receives an input data, of size $\delta_{i-1}$, from the previous stage $S_{i-1}$, performs a computation composed of $w_{i}$ operations, and produces an output data, of size $\delta_{i}$. The computation of a stage is periodically repeated on each input data in the pipeline stream. The input (respectively output) of the stream is initially produced (finally consumed) by an extra stage $S_{0}$ (respectively $S_{n+1}$ ). A graphical representation of a pipelined application is shown in Figure 6.

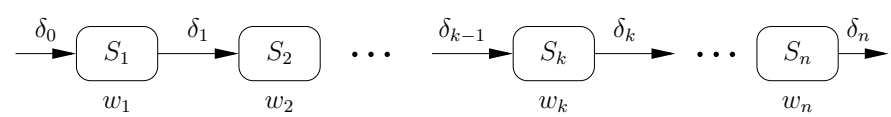

Figure 6: Overview of a pipelined application.

\subsection{Target platform}

The target platform is composed of $p+2$ processors: $p$ computing processors $P_{u}(1 \leq u \leq p)$ are dedicated to host stages $S_{i}(1 \leq i \leq n)$, while $P_{0}$ (also denoted as $P_{i n}$ ) and $P_{p+1}$ (also denoted as $\left.P_{\text {out }}\right)$ are special processors devoted to host the extra stages $S_{0}$ and $S_{n+1}$. Therefore, $P_{\text {in }}$ is dedicated to store initial input data sets of the pipeline and $P_{\text {out }}$ to receive and store the final results. Each processor $P_{u}(1 \leq u \leq p)$ has a speed denoted as $s_{u}$. That means $P_{u}$ takes $X / s_{u}$ time units to execute $X$ operation units. The speed may be identical for all processors $\left(s_{u}=s\right.$ for $\left.1 \leq u \leq p\right)$. In this case, the platform is said to be SpeedHom (homogeneous in speed). On the opposite, the platform is SpeedHet when processors have different speeds.

As shown in Figure 7, all processors are interconnected as a virtual clique. A link between any two processors $P_{u}, P_{v}(0 \leq u, v \leq p+1)$ is bidirectional and has a bandwidth denoted as $b_{u, v}$. Note that a physical link between any processor pair is not required. Instead, the connection of $P_{u}$ to $P_{v}$ may be done through a switch or a path composed of several physical links. In this latter case, $b_{u, v}$ is the bandwidth of the slowest physical link in the path. When the links are identical $\left(b_{u, v}=b\right.$ for all $\left.0 \leq u, v \leq p+1\right)$, the platform is said to be LinkHom.

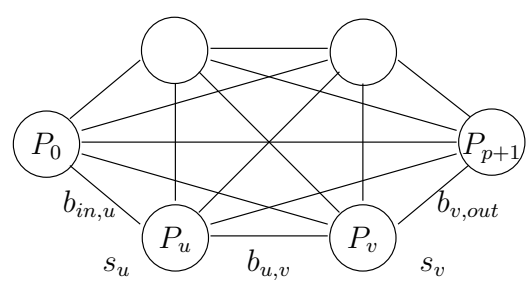

Figure 7: The target platform. 
This is the case for instance in parallel machines. Alternatively, the platform is LinkHet, like in grid infrastructures.

In addition to link bandwidths, the total communication capacity of a processor is limited by its own input/output network card capacity. Formally, we denote by $B_{u}^{i}$ and $B_{u}^{o}$ the input and output card capacity of processor $P_{u}$. Thus, $P_{u}$ cannot receive more than $B_{u}^{i}$ data units nor send more than $B_{u}^{o}$ data units per time unit. When all processors have same card capacities $\left(B_{u}^{i}=B^{i}, B_{u}^{o}=B^{o}\right.$, for all $\left.1 \leq u \leq p\right)$, the platform is said to be CardHom. This is often true when processors are identical (parallel machines, homogeneous clusters). Otherwise, the platform is said to be CardHet.

The platform is assumed to be subject to failures. We consider only fail-silent processor failures without recovering. Thus, a processor can only perform correct actions before eventually crashing (no transient errors). In addition, communication links are assumed to be reliable, hence no data is lost. For the mapping optimization problem, we need to measure the reliability of used processors. This is given by the failure probability $f_{u}\left(0<f_{u}<1\right)$ of each processor $P_{u}(1 \leq u \leq p)$. This failure probability is assumed to be constant, i.e., the same at any time during the execution of a pipelined application. This is because we target a steady-state execution, for instance a scenario with resources loaning/renting. In such a scenario, resources could be suddenly reclaimed by their owners, as during an episode of cycle-stealing $[1,5,11]$. Also, there is no time upper bound for the execution of a streaming application which may involve an arbitrary number of data sets, so the failure probability cannot depend upon execution time. As a consequence, the failure probability should thus be seen as a global indicator of the reliability of a processor. We consider platforms with two failure models. The first model, FailHom, assumes identical failure probabilities for all processors $\left(f_{u}=f\right.$ for all $\left.1 \leq u \leq p\right)$, while the second model, FailHet, assumes different failure probabilities.

Finally, we classify a target platform according to different combinations of processors and links properties. In particular, we consider five classes:

- Fully Homogeneous platforms (FullHom): these platforms are both SpeedHom, CardHom, LinkHom and FailHom.

- Failure Homogeneous platforms: these platforms are FailHom, without any homogeneity constraints on processors speeds, network cards and communication links.

- Speed Heterogeneous platforms: these platforms are SpeedHet, but failures, network cards and communication links are homogeneous.

- Link Heterogeneous platforms: these platforms are LinkHet, but failures, speeds and network cards are homogeneous.

- Failure Heterogeneous platforms: these platforms are FailHet, but speeds, network cards and communication links are homogeneous.

- Fully Heterogeneous platforms (FullHet): these platforms are SpeedHet, CardHet, LinkHet and FailHet.

This classification is relevant from both theoretical and practical perspectives for the mapping optimization problem. Note that Speed Heterogeneous, Link Heterogeneous and Failure Heterogeneous introduce one single level of heterogeneity. We do not consider homogeneous platforms with CardHet, because such platforms have no practical significance. 


\subsection{Communication model}

Communications between processors follow the bounded multi-port model [8]. In this model, multiple communications can take place simultaneously on a same communication link. This assumes the ability to initiate multiple concurrent incoming and outgoing communications, and to share the link bandwidth. This can be done by using multi-threaded communication libraries such as MPICH2 [9]. The bounded characteristic of simultaneous communications is related to the fact that each communication is allotted a bandwidth fraction of the network card, and the sum of these fractions cannot exceed the total capacity of the card. Moreover, we assume full overlap of communications and computations: a stage can simultaneously receive, compute and send independent data. This assumption is reasonable as most state-of-the-art processors are running multi-threaded operating systems capable of such an overlap.

\subsection{Mapping problem}

Mapping a pipelined application is the process of allocating target execution processors to the pipeline stages. To decide how application stages are assigned to processors, different rules may be adopted. For instance, in one-to-one mappings, each stage is assigned to a distinct processor, and thus each processor processes only one single stage. A less restrictive class of mappings, interval mappings, are such that a processor may be processing a consecutive subset of stages. In this report, we focus on such interval mappings, which have been widely studied $[3,4,12,13]$.

In the following, we formally define interval mappings and the adopted replication model to deal with processors failures. Then, we express the period and failure probability of a pipelined application, once given a mapping.

Interval mappings: in an interval mapping (with replication), stages $S_{i}(1 \leq i \leq n)$ are partitioned into $m \leq n$ intervals, and each interval is assigned to a distinct set of processors. This consists in partitioning the interval of stages indices $[1 . . n]$ into $m$ intervals $I_{j}=\left[d_{j}, e_{j}\right]$, where $d_{j} \leq e_{j}$ for $1 \leq j \leq m, d_{1}=1, d_{j+1}=e_{j}+1$ for $1 \leq j \leq m-1$ and $e_{m}=n$. Each interval $I_{j}$ is mapped to one set of processors whose indices belong to alloc $\left(d_{j}\right)$. In such a mapping, $\operatorname{alloc}(i)=\operatorname{alloc}\left(d_{j}\right)$ for $d_{j} \leq i \leq e_{j}$. In addition, a processor cannot process two distinct intervals, i.e., alloc $\left(d_{j}\right) \cap$ alloc $\left(d_{j^{\prime}}\right)=\emptyset$ for $1 \leq j, j^{\prime} \leq m, j \neq j^{\prime}$.

Replication model: as discussed in Section 3.2, processors are subject to failures. To deal with such failures, we adopt an active replication protocol. In more details, all processors $P_{u}$ $\left(u \in \operatorname{alloc}\left(d_{j}\right), 1 \leq j \leq m\right)$ perform the same assigned interval computations (active) on the same input data. Therefore, the output data of an interval $I_{j}$ has to be sent to all processors $P_{v}$ with $v \in \operatorname{alloc}\left(d_{j+1}\right)$. To avoid redundant input data, a consensus protocol [14] is executed by surviving processors $P_{u}\left(u \in\right.$ alloc $\left.\left(d_{j}\right)\right)$ after each execution of interval $I_{j}$ on an input data. The consensus aims at electing a processor as the sole one that will send the output data of $I_{j}$ to all surviving processors $P_{v}$. This protocol is illustrated in Figure 8, where all processors are surviving. It allows to pay at most $\mid$ alloc $\left(d_{j+1}\right) \mid$ outgoing communications by the elected processor (according to the bounded multi-port communication model) and only one incoming communication by $P_{v}$. In the scope of this report, we assume that communications intrinsic to the consensus have a negligible overhead. Hence, only the multiple outgoing communications executed by an elected processor are accounted for in the performance model. 


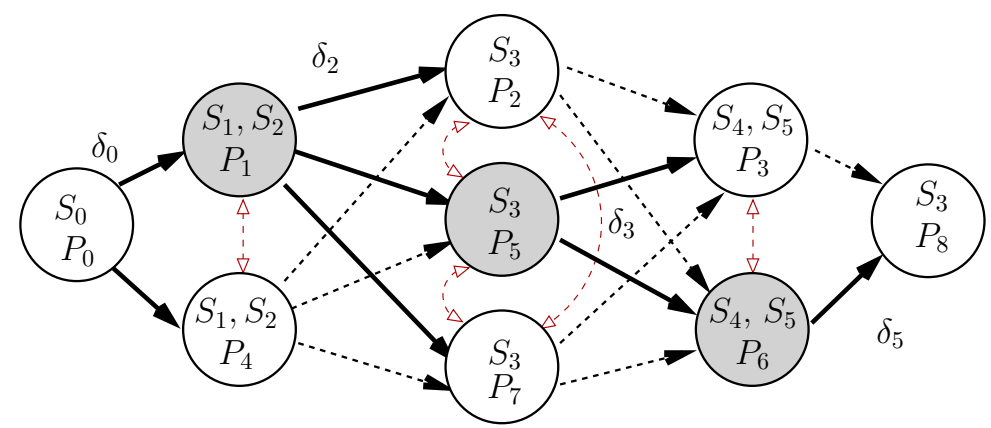

Figure 8: Replication model. Each processor periodically receives input data from one predecessor (on the plain incoming arrow), executes all assigned interval stages, exchanges extra messages (on dashed vertical arrows) with processors allocated to the same interval, agrees upon which processor (filled circle) has to send the result to all its successors (on plain outgoing arrows).

Period: the period $\mathcal{P}$ of an interval mapping with replication on the most general FullHet platforms is expressed as:

$$
\mathcal{P}=\max _{1 \leq j \leq m} \max _{u \in \text { alloc }\left(d_{j}\right)} \max \left\{\frac{\delta_{d_{j}-1}}{\min _{v \in \operatorname{alloc}\left(d_{j}-1\right)}\left(b_{v, u}, B_{u}^{i}\right)}, \frac{\sum_{i=d_{j}}^{e_{j}} w_{i}}{s_{u}}, \frac{\delta_{e_{j}}}{\min _{v \in \text { alloc }\left(d_{j+1}\right)}\left(b_{u, v}\right)}, \frac{\left|\operatorname{alloc}\left(d_{j+1}\right)\right| \delta_{e_{j}}}{B_{u}^{o}}\right\} .
$$

This formula considers the worst case scenario, where only one processor $P_{u}$ allocated to interval $I_{j}\left(u \in \operatorname{alloc}\left(d_{j}\right)\right)$ is surviving, while all processors allocated to the next interval $I_{j+1}$ are alive. The formula for $P_{u}$ accounts for input data (one communication from the slowest processor assigned to interval $I_{j-1}$, hence the minimum taken on link and network card bandwidths), for computations, and for output data (constraint on each communication link, on the network card, and there is a total of $\mid$ alloc $\left(d_{j+1}\right) \mid$ communications). There remains to take the maximum of these cycle times for $u \in \operatorname{alloc}\left(d_{j}\right)$, and then a global maximum over all intervals.

Failure probability: the failure probability $\mathcal{F}$ of a pipelined application in the most general situation (FailHet) is computed by the following formula:

$$
\mathcal{F}=1-\prod_{1 \leq j \leq m}\left(1-\prod_{u \in \text { alloc }\left(d_{j}\right)} f_{u}\right) .
$$

This formula is obtained from the fact that the execution of an application is successful if and only if there remains at least one surviving processor per set alloc $\left(d_{j}\right)$ of processors allocated to each interval of stages, i.e., for $1 \leq j \leq m$.

Finally, the target optimization problem is to determine the best interval mapping that minimizes the failure probability $\mathcal{F}$ given a threshold period $P_{\text {max }}$ : the mapping must be such that $\mathcal{P} \leq P_{\text {max }}$. 


\section{Complexity results}

We first prove that the problem can be solved in polynomial time on a fully homogeneous (FullHom) platform (SpeedHom, LinkHom, CardHom and FailHom), using dynamic programming. However, the problem becomes NP-hard as soon as we add one level of heterogeneity (SpeedHet, LinkHet or FailHet). Recall that we do not consider homogeneous platforms with CardHet, because such platforms have no practical significance.

Theorem 1. For fully homogeneous platforms, the optimal interval mapping which minimizes the failure probability under a fixed period threshold can be determined in polynomial time $O\left(n^{2} p^{3}\right)$.

Proof. We exhibit here a dynamic programming algorithm which computes the optimal mapping. Let $\mathcal{P}$ denote the threshold period. We recursively compute the value of $R\left(i, q, q_{\text {succ }}\right)$, which is the optimal value of reliability, i.e., $1-\mathcal{F}$, that can be achieved by any interval mapping of stages $S_{1}$ to $S_{i}$, using exactly $q$ processors, and given that the following interval (starting with stage $S_{i+1}$ ) is using exactly $q_{\text {succ }}$ processors. The goal is to determine $\max _{1 \leq q \leq p} R(n, q, 1)$, since the final interval has only one succeeding processor, $P_{\text {out }}$. Maximizing the reliability amounts to minimizing the failure probability. The recurrence relation can be expressed as:

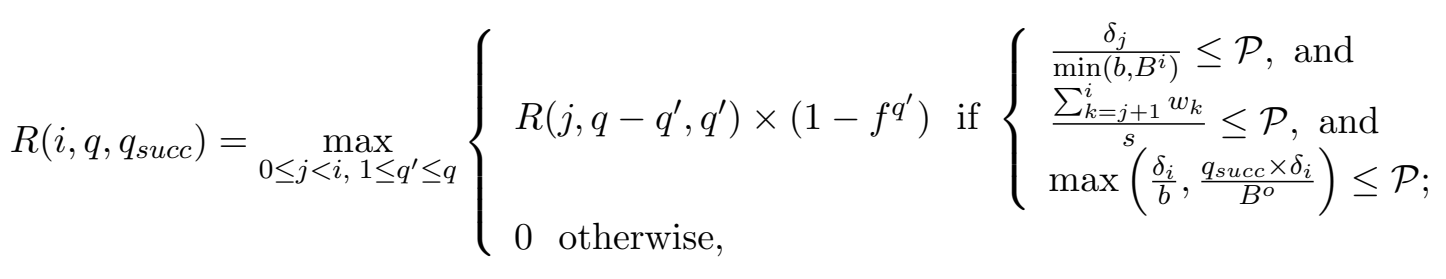

for $1 \leq i \leq n, 1 \leq q, q_{\text {succ }} \leq p$, with the initialization

$$
\begin{gathered}
R\left(0, q, q_{\text {succ }}\right)=1 \text { for } 1 \leq q, q_{\text {succ }} \leq p, \\
R\left(i, 0, q_{\text {succ }}\right)=0 \text { for } 1 \leq i \leq n, 1 \leq q_{\text {succ }} \leq p .
\end{gathered}
$$

The recurrence is easy to justify: to compute $R\left(i, q, q_{\text {succ }}\right)$, we create an interval from stages $S_{j+1}$ to $S_{i}$, for $0 \leq j<i$, and we allocate it onto $q^{\prime}$ processors. Since everything is homogeneous, the contribution to the reliability from this interval is $\left(1-f^{q^{\prime}}\right)$, and the reliability is the product of reliabilities of all intervals. The solution is valid only if the constraint on the period is satisfied, hence the condition checking whether input communications, computations and output communications satisfy the bound. The parameter $q_{s u c c}$ is needed to compute the time required by output communications. In the recursive call, the new value of $q_{\text {succ }}$ is thus $q^{\prime}$, and we consider stages $S_{1}$ to $S_{j}$.

For the initialization, if we have already mapped all stages, the contribution to the reliability is $1\left(R\left(0, q, q_{\text {succ }}\right)\right)$, while if we used all processors and we still have stages that remain not allocated, then the reliability is set to 0 in order to indicate that this solution is not valid (the failure probability becomes 1 ).

The complexity of this dynamic programming algorithm is bounded by $O\left(n^{2} p^{3}\right)$ : we need to compute $O\left(n p^{2}\right)$ values of $R\left(i, q, q_{\text {succ }}\right)$, and it takes a time $O(n p)$ to compute one value in the worst case (maximum over $j$ and $q^{\prime}$ ). 
Theorem 2. For SpeedHet, LinkHet or FailHet platforms, finding the optimal interval mapping which minimizes the failure probability under a fixed period threshold is NP-hard (even with no communication cost for SpeedHet and FailHet, and with no replication for LinkHet).

Proof. We consider the associated decision problem INT-PF: given a period $P$ and a failure probability $F$, is there a mapping of period less than $P$ and of failure probability less than $F$ ?

It is clear that INT-PF is in NP: given a period, a failure probability and a mapping, it is easy to check in polynomial time that it is valid by computing its period and failure probability.

The completeness for SpeedHet platforms comes directly from [3], in which we prove that minimizing the period with different speed processors is NP-hard (this is the heterogeneous chains-on-chains problem, denoted CoC-HET). Thus, starting from an instance of CoC-HET, we create an instance of INT-PF with no communication, $F$ set to 1 , and the same period as in CoC-HET. There is no need to replicate in this case: it can only decrease the period, and we do not care about failures since $F=1$. The two problems are thus fully identical.

To establish the completeness for LinkHet platforms, we use a reduction from the Bottleneck Traveling Salesman (BTS) problem [7]. We consider an instance $\mathcal{I}_{1}$ of BTS: given a graph $G$ with $m+2$ nodes labeled $v_{\text {in }}, v_{\text {out }}$, and $v_{i}(1 \leq i \leq m)$, positive edge weights $d\left(v_{i}, v_{j}\right)$, and a bound $K$, does there exist a Hamiltonian path from $v_{i n}$ to $v_{\text {out }}$ in the graph whose largest edge weight does not exceed $K$ ?

We build the following instance $\mathcal{I}_{2}$ of our problem: the pipeline is composed of $n=m$ stages with $w=1 / K$ and $\delta=1$; the platform is made of $p=n$ computing processors $P_{i}$ with speeds $s=1$, network cards $B^{i}=B^{o}=1 / \min _{i \neq j}\left(d\left(v_{i}, v_{j}\right)\right)$, and failure probabilities 0 (fully reliable processors). The interconnection graph between processors is exactly $G$, with $P_{\text {in }}$ mapped on $v_{\text {in }}$ and $P_{\text {out }}$ mapped on $v_{\text {out }}$. Bandwidths are given by $b_{i, j}=1 / d\left(v_{i}, v_{j}\right)$. We then set $P=1 / K$ and $F=0$ (in fact, we tackle the period minimization problem since all processors are assumed to be fully reliable).

For the period to be respected, we cannot have more than one stage per processor: we necessarily end up with a one-to-one mapping without replication. Hence all communication costs are paid, and the period is respected if and only if we can find a Hamiltonian cycle, i.e., if and only if instance $\mathcal{I}_{1}$ has a solution.

To establish the completeness for FailHet platforms, we use a reduction from 3-PARTITION [7], which is NP-complete in the strong sense. We consider an instance $\mathcal{I}_{1}$ of 3-PARTITION: given a set $\left\{a_{1}, \ldots, a_{3 m}\right\}$ of $3 m$ integers, and an integer $B$ such that $\sum_{1<j<3 m} a_{j}=m B$, does there exist $m$ independent subsets $B_{1}, \cdots, B_{m}$ of $\{1, \cdots, 3 m\}$ such that for all $1 \leq i \leq m, \sum_{j \in B_{i}} a_{j}=B$ ?

We build the following instance $\mathcal{I}_{2}$ of our problem (with no communication cost): the pipeline is composed of $n=m$ stages with $w=1$, and $p=3 m$ processors with speeds $s=1$, and failure probabilities $f_{u}=2^{-a_{u}}$, for $1 \leq u \leq p$. We then set $P=1$ and $F=1-\left(1-2^{-B}\right)^{m}$.

Note that the size of $\mathcal{I}_{2}$ is polynomial in the size of $\mathcal{I}_{1}$. Indeed, since 3-PARTITION is NP-complete in the strong sense, we could encode $\mathcal{I}_{1}$ in unary, and thus the size of the instance would be in $O(m B)$. Moreover, the values of $f_{u}$ and $F$ can be encoded in binary and thus their size is polynomial in the size of $\mathcal{I}_{1}$.

Now we show that $\mathcal{I}_{1}$ has a solution if and only if $\mathcal{I}_{2}$ has a solution. Suppose first that $\mathcal{I}_{1}$ has a solution. For $1 \leq i \leq m$, stage $S_{i}$ is mapped onto the processors of subset $B_{i}$, 
thus respecting the period of 1 , and this stage is successful with a probability $1-\prod_{j \in B_{i}} f_{j}=$ $1-\prod_{j \in B_{i}} 2^{-a_{j}}=1-2^{\sum_{j \in B_{i}}-a_{j}}=1-2^{-B}$. Since there are $m$ intervals of one stage in the mapping, the total failure probability is $1-\left(1-2^{-B}\right)^{m}$, which means that $\mathcal{I}_{2}$ has a solution.

Suppose now that $\mathcal{I}_{2}$ has a solution. A processor cannot handle more than one stage, otherwise its period becomes greater than $P=1$ (it would be at least $2 w / s=2$ ). Let $T_{i}$ be the set of indices of processors working onto stage $S_{i}$, for $1 \leq i \leq m$. The failure probability is thus $1-\prod_{i=1}^{m}\left(1-2^{-\sum_{j \in T_{i}} a_{j}}\right)$. This quantity is always strictly larger than $1-\prod_{i=1}^{m}\left(1-2^{-B}\right)$ unless when $\sum_{j \in T_{i}} a_{j}=B$ for $1 \leq i \leq m$, as was proved by Lemma 2 in [2]. Thus, the processor indices of the mapping correspond to a solution of $\mathcal{I}_{1}$, which concludes the proof.

\section{Mixed integer linear program formulation}

This section deals with the problem of maximizing reliability under period constraints. As stated in Section 4, this problem is NP-hard for interval mappings on heterogeneous platforms. In this section, we introduce a mixed integer linear program which computes the optimal interval mapping on such platforms, but restricting to FailHom processors.

We failed to derive a program with a polynomial number of variables for FailHet platforms. The reason can be seen from Equation 2: we would have needed to create a variable for all possible processor subsets $S$, and to pre-compute the corresponding product $\prod_{u \in S} f_{u}$. Instead, with FailHom platforms, we succeed to keep a polynomial number of variables; the key observation is that we only need to record the number of processors assigned to each interval.

Recall that a pipelined application is composed of $n$ stages and the target platform of $p$ processors, plus two fictitious extra stages $S_{0}$ and $S_{n+1}$ respectively assigned to two extra processors $P_{0}$ and $P_{p+1}$. We start by defining the program parameters and variables, then we describe the linear constraints of the problem:

\section{Parameters:}

- $n$ : number of application stages, except $S_{0}, S_{n+1}$.

- $p$ : number of target platform processors, except $P_{0}, P_{p+1}$.

- $\delta_{i}(i \in[0 . . n])$ : the size of output data of stage $S_{i}$.

- $w_{i}(i \in[1 . . n])$ : the workload of stage $S_{i}$.

- $s_{u}(u \in[1 . . p])$ : the speed of processor $P_{u}$.

- $B_{u}^{i}(u \in[1 . . p])$ : the input network card capacity of processor $P_{u}$.

- $B_{u}^{o}(u \in[1 . . p])$ : the output network card capacity of processor $P_{u}$.

- $b_{u, v}(u, v \in[0 . . p+1], u \neq v)$ : the bandwidth of link $P_{u} \leftrightarrow P_{v}$.

- $\Lambda_{k}(k \in[1 . . p])$ : double equal to $\log \left(1-f^{k}\right)$, where $f$ is the failure probability of all processors (FailHom platform).

- $P_{\max }$ : the constrained maximum period. 


\section{Decision variables:}

- $R_{\log }$ : the logarithm of the reliability probability to maximize.

- $x_{i, u}(i \in[0 . . n+1], u \in[0 . . p+1])$ : a boolean variable equal to 1 if stage $S_{i}$ is assigned to processor $P_{u}$. Hypothesis: $x_{0,0}=x_{n+1, p+1}=1, x_{i, 0}=0$ for $i \geq 1, x_{0, u}=0$ for $u \geq 1$, $x_{i, p+1}=0$ for $i \leq n$ and $x_{n+1, u}=0$ for $u \leq p$.

- $y_{i}(i \in[0 . . n])$ : a boolean variable equal to 0 if stages $S_{i}$ and $S_{i+1}$ belong to a same interval. Hypothesis: $y_{0}=y_{n}=1$.

- $z_{i, u, v}(i \in[0 . . n], u, v \in[0 . . p+1])$ : a boolean variable equal to 1 if stage $S_{i}$ is assigned to $P_{u}$ and stage $S_{i+1}$ is assigned to $P_{v}$. When $u \neq v, S_{i}$ is not assigned to $P_{v}$ and $S_{i+1}$ is not assigned to $P_{u}\left(S_{i}\right.$ and $S_{i+1}$ are in distinct intervals). Hypothesis: $z_{i, 0, v}=0$ for $i \neq 0$ and all $v$, and $z_{i, u, p+1}=0$ for $i \neq n$ and all $u$.

- first $_{u}$ and last $_{u}(u \in[1 . . p])$ : integer variables which denotes, in order, the first and last stages $S_{\text {first }}$ and $S_{\text {last }_{u}}$ assigned to processor $P_{u}$. Thus $P_{u}$ is assigned the interval $\left[\right.$ first $_{u}$, last $\left._{u}\right]$. Hypothesis: $1 \leq$ first $_{u} \leq$ last $_{u} \leq n$.

- $n b P_{i}(i \in[0 . . n+1])$ : integer variable which denotes the number of processors allocated to stage $S_{i}$. Hypothesis: $1 \leq n b P_{i} \leq p$ and $n b P_{0}=n b P_{n+1}=1$.

- PperINT $T_{i, k}(i \in[1 . . n], k \in[1 . . p])$ : boolean variable equal to 1 if stages $S_{i}$ and $S_{i+1}$ are assigned to different processors and if $S_{i}$ is assigned to exactly $k$ processors.

\section{Constraints:}

- Stages assignment on processors:

- If stage $S_{i}$ is assigned to $P_{u}$ and not to $P_{v}$ and stage $S_{i+1}$ is assigned to $P_{v}$ and not to $P_{u}$, then $z_{i, u, v}=1$ :

$$
\forall i \in[0 . . n], \forall u, v \in[0 . . p+1], u \neq v, \quad x_{i, u}+x_{i+1, v}+\left(1-x_{i, v}\right) \leq 2+z_{i, u, v}
$$

- If stages $S_{i}$ and $S_{i+1}$ are both assigned to $P_{u}$, then $z_{i, u, u}=1$ :

$$
\forall i \in[0 . . n], \forall u \in[0 . . p+1], \quad x_{i, u}+x_{i+1, u} \leq 1+z_{i, u, u}
$$

- If $z_{i, u, v}=1$, then stage $S_{i}$ is assigned to $P_{u}$ and stage $S_{i+1}$ to $P_{v}$. In addition, when $u \neq v, S_{i}$ is not assigned to $P_{v}$ nor $S_{i+1}$ to $P_{u}$ :

$$
\begin{aligned}
\forall i \in[0 . . n], \forall u, v \in[0 . . p+1], & & z_{i, u, v} \leq x_{i, u} \\
\forall i \in[0 . . n], \forall u, v \in[0 . . p+1], & & z_{i, u, v} \leq x_{i+1, v} \\
\forall i \in[0 . . n], \forall u, v \in[0 . . p+1], u \neq v, & & z_{i, u, v} \leq 1-x_{i, v}
\end{aligned}
$$

- If stages $S_{i}$ and $S_{i+1}$ are both assigned to $P_{u}$, then $y_{i}=0$ :

$$
\forall i \in[0 . . n], \quad \sum_{u \in[0 . . p+1]} \sum_{v \in[0 . . p+1], u \neq v} z_{i, u, v} \geq y_{i}
$$


- If stages $S_{i}$ and $S_{i+1}$ are assigned to different processors, then $y_{i}=1$ :

$$
\forall i \in[0 . . n], \forall u \in[0 . . p+1], \forall v \in[0 . . p+1], u \neq v, \quad z_{i, u, v} \leq y_{i}
$$

- If stage $S_{i}$ is assigned to $P_{u}$, then $z_{i, u, u}$ is the inverse of $y_{i}$ :

$$
\begin{array}{ll}
\forall i \in[0 . . n], \forall u \in[1 . . p], & z_{i, u, u} \leq 1-y_{i} \\
\forall i \in[0 . . n], \forall u \in[1 . . p], & x_{i, u}-z_{i, u, u} \leq y_{i}
\end{array}
$$

- The bounds of an interval:

- If stage $S_{i}$ is assigned to $P_{u}$, then first $_{u} \leq i \leq$ last $_{u}$ :

$$
\begin{array}{ll}
\forall i \in[1 . . n], \forall u \in[1 . . p], & \text { first }_{u} \leq i * x_{i, u}+n *\left(1-x_{i, u}\right) \\
\forall i \in[1 . . n], \forall u \in[1 . . p], & \text { last }_{u} \geq i * x_{i, u}
\end{array}
$$

- If stage $S_{i}$ is assigned to $P_{u}$ and stage $S_{i+1}$ to $P_{v}(v \neq u)$, i.e., $z_{i, u, v}=1$, then last $_{u} \leq i$ and first $_{v} \geq i+1$ since we consider intervals:

$\forall i \in[1 . . n-1], \forall u \in[1 . . p], \forall v \in[1 . . p], v \neq u, \quad$ last $_{u} \leq i * z_{i, u, v}+n *\left(1-z_{i, u, v}\right)$

$\forall i \in[1 . . n-1], \forall u \in[1 . . p], \forall v \in[1 . . p], v \neq u, \quad$ first $_{v} \geq(i+1) * z_{i, u, v}$

- If a processor $P_{u}$ is not used, then last $_{u}$ and first $t_{u}$ are forced to be equal to 1 :

$$
\begin{array}{ll}
\forall u \in[1 . . p], & \text { first }_{u} \leq \sum_{i \in[1 . . n]} x_{i, u} * n+1 \\
\forall u \in[1 . . p], & \text { last }_{u} \leq \sum_{i \in[1 . . n]} x_{i, u} * n+1
\end{array}
$$

- The number of processors allocated to a stage/interval:

- Each stage is assigned exactly $n b P_{i}$ processors:

$$
\forall i \in[0 . . n+1], \sum_{u \in[0 . . p+1]} x_{i, u}=n b P_{i}
$$

- If stages $S_{i}$ and $S_{i+1}$ are both assigned to a same processor, then $n b P_{i}=n b P_{i+1}$ :

$$
\begin{array}{ll}
\forall i \in[0 . . n], & n b P_{i}-n b P_{i+1} \leq y_{i} * p \\
\forall i \in[0 . . n], & n b P_{i}-n b P_{i+1} \geq-y_{i} * p
\end{array}
$$

- If $y_{i}=1$, then exactly one value of $k \in[1 . . p]$ corresponds to the number of processors allocated to $S_{i}$, in which case, $\operatorname{Pper} I N T_{i, k}=1$, otherwise $\operatorname{PperINT_{i,k}=}$ 0 for all $i$ and all $k$ :

$$
\forall i \in[1 . . n], \quad \sum_{k \in[1 . . p]} \operatorname{PperINT} T_{i, k}=y_{i}
$$


- If PperINT $T_{i, k}=1$, then $k=n b P_{i}$ :

$$
\begin{array}{ll}
\forall i \in[1 . . n], & n b P_{i}-\sum_{k \in[1 . . p]} k * P \operatorname{PerIN} T_{i, k} \leq\left(1-y_{i}\right) * p \\
\forall i \in[1 . . n], & n b P_{i}-\sum_{k \in[1 . . p]} k * \operatorname{PperINT} T_{i, k} \geq\left(1-y_{i}\right)
\end{array}
$$

- Cycle-time of a processor:

- The workload of a processor is expressed as:

$$
\forall u \in[1 . . p], \quad \sum_{i \in[1 . . n]} \frac{w_{i}}{s_{u}} x_{i, u} \leq P_{\max }
$$

- Incoming communications ${ }^{1}$ of a processor are expressed as:

$$
\begin{aligned}
& \forall u \in[1 . . p], \forall t \in[0 . . p], t \neq u, \quad \sum_{i \in[1 . . n]} \frac{\delta_{i-1}}{B_{u}^{i}} z_{i-1, t, u} \leq P_{\max } \\
& \forall u \in[1 . . p], \forall t \in[0 . . p], t \neq u, \quad \sum_{i \in[1 . . n]} \frac{\delta_{i-1}}{b_{t, u}} z_{i-1, t, u} \leq P_{\max }
\end{aligned}
$$

- Outgoing communications ${ }^{1}$ of a processor are expressed as:

$$
\begin{aligned}
& \forall u \in[1 . . p], \sum_{i \in[1 . . n]} \sum_{v \in[1 . . p+1], v \neq u} \frac{\delta_{i}}{B_{u}^{o}} z_{i, u, v} \leq P_{\max } \\
& \forall u \in[1 . . p], \forall v \in[1 . . p+1], v \neq u, \quad \sum_{i \in[1 . . n]} \frac{\delta_{i}}{b_{u, v}} z_{i, u, v} \leq P_{\max }
\end{aligned}
$$

- Failure probability: the reliability probability (inverse of failure probability) of the whole pipelined application is expressed as:

$$
\sum_{i \in[1 . . n]} \sum_{k \in[1 . . p]} \Lambda_{k} * \operatorname{Pper} I N T_{i, k} \geq R_{\log }
$$

Objective function: we aim at finding values for each variable in order to maximize $R_{\text {log }}$, given that all constraints are satisfied.

\footnotetext{
${ }^{1}$ Recall that communications follow the multi-port model with overlap.
} 


\section{Heuristics}

In this section, we propose heuristics for interval mappings with replication on FullHet platforms (Section 3.2). The objective is to find a mapping optimizing the failure probability $\mathcal{F}$ under a fixed period bound $P_{\max }$. From Section 4, we know that determining the optimal mapping is NP-hard on heterogeneous platforms. In addition, we know that to satisfy the period bound, it can be necessary to have multiple intervals. Therefore, we propose a large number of stage partitioning techniques to find a good mapping. In particular, we propose two classes of heuristics. In the first class, the partitioning and mapping phases are addressed by different procedures (Section 6.1). In the second class, partitioning and mapping decisions are made on the fly as the heuristics progress (Section 6.2). We intend to explore quite a comprehensive set of mapping solutions, thereby (hopefully) producing a final mapping with satisfyingly small failure probability.

Before presenting the heuristics, we discuss how the allocation of a processor to an interval succeeds during the mapping process.

Processor allocation: during the processor mapping phase, the cycle-time of a candidate processor for assignment to an interval $I_{j}=\left[d_{j}, e_{j}\right](1 \leq j \leq m \leq n)$ is computed to verify the upper fixed period bound $P_{\max }$. According to Formula 1 (computation of the period $P$ of a mapping), this cycle-time depends on the processors allocated to both previous and next intervals $\left(I_{j-1}, I_{j+1}\right)$, if they exist. In addition, allocating a processor to an interval changes the cycle-time of these processors and eventually the period of the whole application. However, when attempting an allocation, some intervals may be not yet assigned. Hence, we introduce some rules to be able to return a cycle-time value at each moment during the mapping. These rules are defined through Algorithm 1. This algorithm checks whether a processor may be allocated to a given interval, i.e., preserves $\mathcal{P} \leq P_{\max }$. This verification is essential for the success of the progressive mapping done by the proposed heuristics. In the algorithm, the notation $I_{0}$ (respectively $I_{m+1}$ ) is used to design the interval composed of the extra stage $S_{0}$ (respectively $\left.S_{n+1}\right)$. The set alloc $\left(I_{j}\right)(1 \leq j \leq m)$ contains the processors allocated to interval $I_{j}$ before the current application of Algorithm 1.

\subsection{Class 1: Heuristics partitioning then mapping}

The heuristics presented in this section (Class 1) work in two phases. First, the application is partitioned into a set of intervals. Then we try to map these intervals onto the platform in order to satisfy the bound on the period, and we compute the reliability of the mapping. We try several different partitionings, and keep the solution which returns the most reliable mapping.

\subsubsection{Partitioning phase}

We start with the partitioning phase. Different partitions are returned, by varying the number of target intervals for the partition: the stages are partitioned into $k$ intervals, with $1 \leq k \leq$ $\min (n, p)$. Three criteria are considered for the partitioning phase.

- Communication cost: stages are split at the $(k-1)$ smallest output data sizes $\left(\delta_{i}\right)$. Then, longest inter-stage communications are avoided and replaced by local memory 


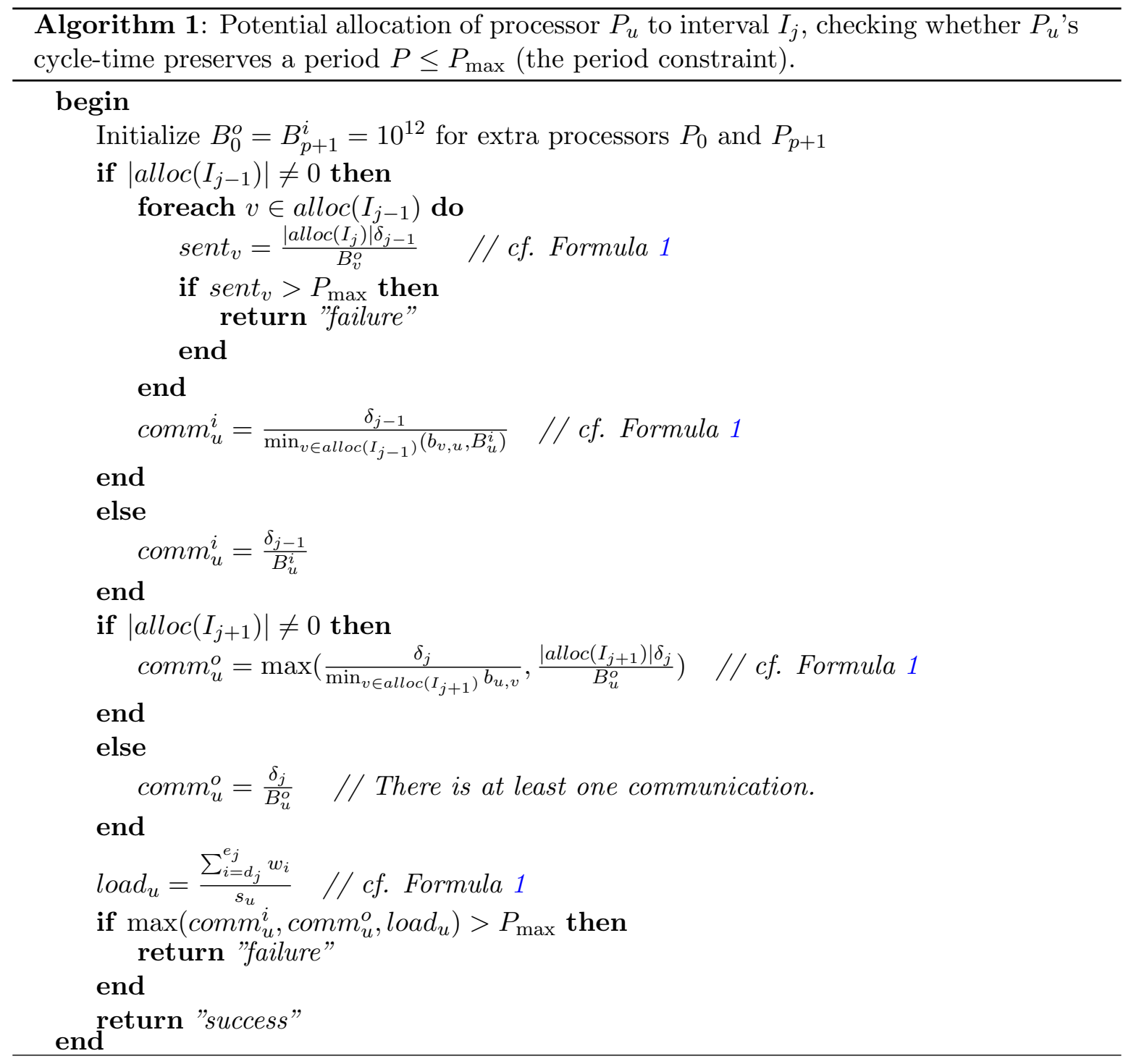


accesses on a processor. In the rest of this report, we identify heuristics using this split criteria by a prefix Partc.

- Computation cost: stages are split into $k$ intervals such that the computation load of each interval approximates the average $\frac{\sum_{i=1}^{n} w_{i}}{k}$. Then, costly intervals in terms of computation may be reduced. In the rest of this report, we identify heuristics using this split criteria by a prefix Partw.

- Random partitioning: stages are split into $k$ intervals at a random place. In the rest of this report, we identify heuristics using this split criteria by a prefix Partr.

These three ways of interval creation seem to be a good trade-off when mapping computation costly, and/or communication costly, stages on various platforms (homogeneous or heterogeneous).

\subsubsection{Heuristics for mapping pre-defined intervals}

This section presents four main heuristics and derives some variants. These heuristics differ in the way processors are distributed over the pre-defined intervals (computed with one of the previous split criteria), and in the priority to order the assignment of these intervals.

Small: smallest $f_{u}$ - This greedy heuristic starts by randomly assigning each interval to one processor satisfying the period constraint. Then, it repeatedly assigns the interval with the highest failure probability to the more reliable processor. As soon as a processor is allocated to an interval, it cannot be reused any more. After all processors are considered, the heuristic attempts to improve the global failure probability. For that, it repeatedly merges the interval with the highest failure probability with previous or next intervals (Algorithm 6). The merge process is done as long as the failure probability can be decreased and the period bound is still satisfied. The heuristic is further detailed in Algorithm 2.

Snake: snake allocation of processors - This heuristic assigns each interval to the most reliable processor satisfying the period constraint. At the next step, each interval is assigned to the least reliable processor, and steps are alternated. As soon as a processor is allocated to an interval, it cannot be reused any more. After all intervals/processors are treated, the heuristic attempts to improve the failure probability of the resulting mapping. For that, it performs the same merge step as done by the Small heuristic (application of Algorithm 6). The heuristic is further detailed in Algorithm 3. From this heuristic, we can derive some variants, depending upon the order in which intervals are considered for assignment. In the present work, we define two variants Snake-c and Snake-w. Snake-c considers intervals in a decreasing order of their output data size $\left(\delta_{e_{j}}\right)$, while Snake-w considers intervals in a decreasing order of their workload $\left(\sum_{i=d_{j}}^{e_{j}} w_{i}\right)$. Therefore, the mapping priority is given to costly intervals in terms of either their output communications or their workload.

BCT: biggest cycle-time - this heuristic repeatedly considers each interval and searches the most critical processor, i.e., with the longest cycle-time satisfying the period $P_{\max }$, and allocates it to this interval. As soon as a processor is allocated to an interval, it cannot be reused any more. After all intervals/processors are treated, the heuristic attempts to 

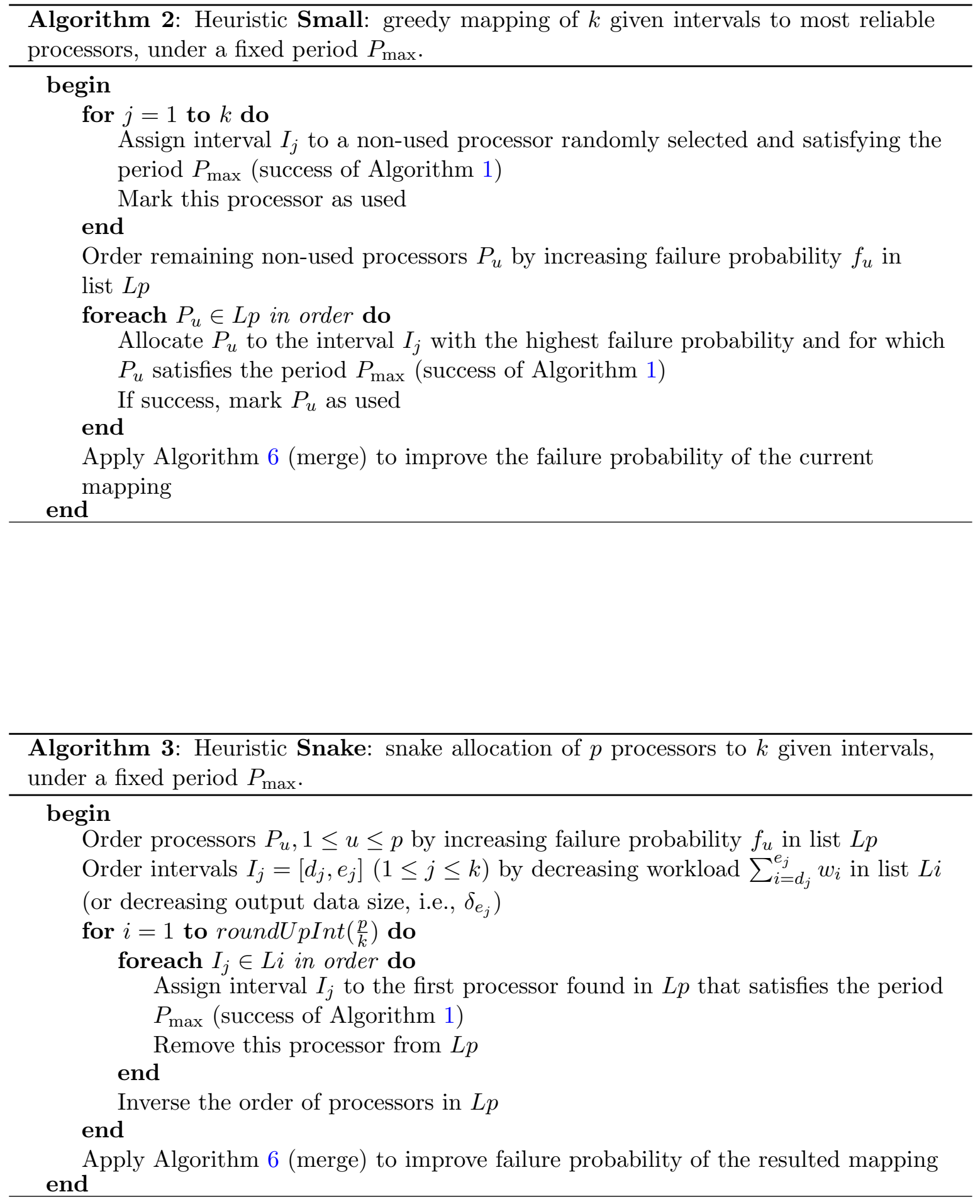
improve the failure probability of the computed mapping, with Algorithm 6 similarly to previous heuristics. The BCT heuristic is further detailed in Algorithm 4. We can also derive some variants, depending upon the order in which intervals are treated. As for the Snake heuristic, we define two variants BCT-c and BCT-w. BCT-c (respectively BCT-w) considers intervals in a decreasing order of their output data size (resp. their workload). We recall that the objective of such variants is to give a priority for mapping costly intervals.

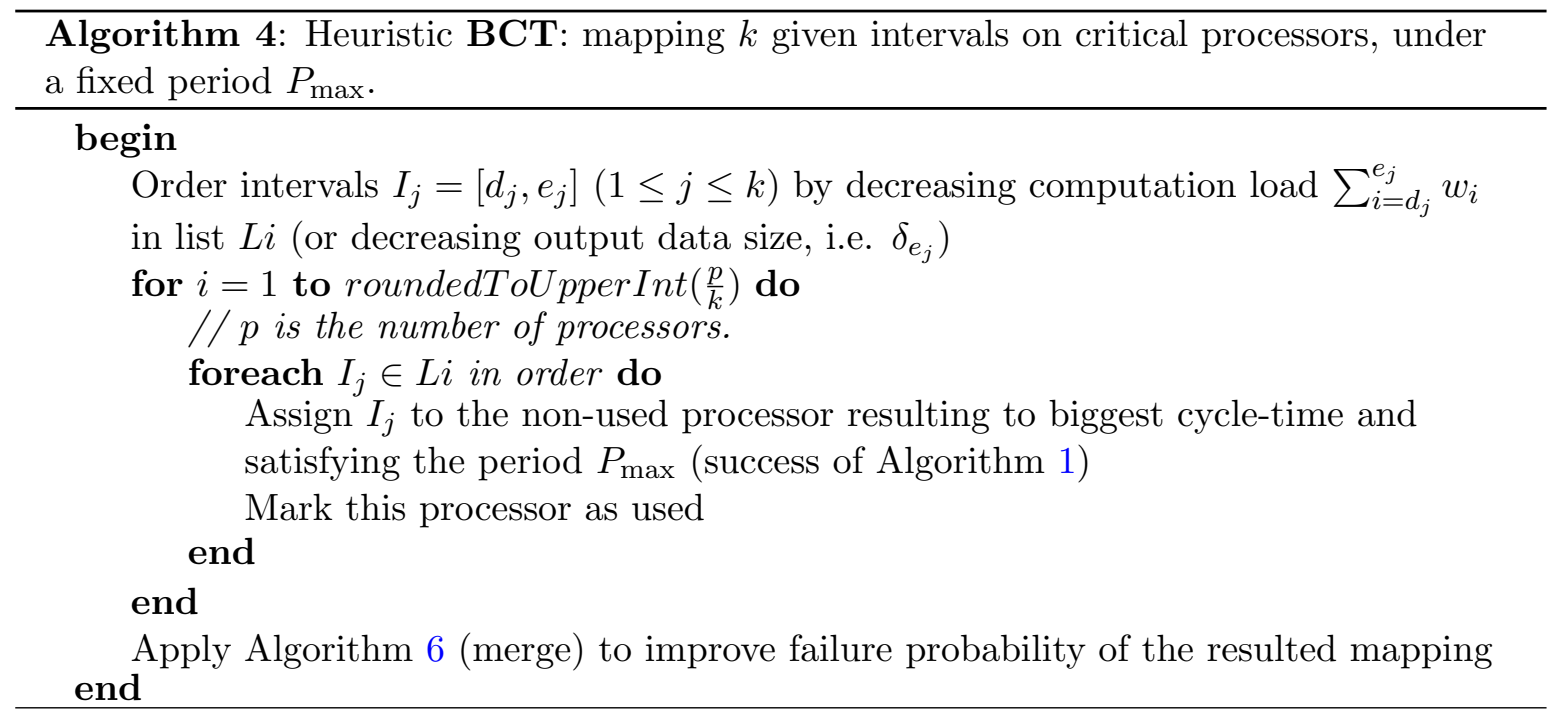

Bal: balancing failure probabilities - This heuristic assigns each interval $I_{j}$ to a set of most critical processors, i.e., with the longest cycle-time satisfying the period $P_{\max }$. This set $\left(\operatorname{alloc}\left(I_{j}\right)\right)$ is such that the product of the processors failure probabilities $\prod_{u \in \text { alloc }\left(I_{j}\right)} f_{u}$ approximates the average value $\sqrt[k]{\prod_{u \in[1 . . p]} f_{u}}$ ( $p$ is the number of all processors). As soon as a processor is allocated to an interval, it can not be reused any more. When all intervals are assigned, the heuristic attempts to improve the failure probability of the computed mapping by applying the intervals merging algorithm (Algorithm 6). The heuristic is further detailed in Algorithm 5. As for Snake and BCT heuristics, we define two variants of the Bal one: Bal-c and Bal-w. Bal-c (respectively Bal-w) treats the intervals in a decreasing order of their output data size (resp. their workload). The objective is still the same, i.e., to provide a mapping priority for costly intervals.

\subsubsection{Partitioning-then-mapping heuristics}

Given a partitioning criteria, we map the intervals of a partition using one of the mapping heuristics. With three different partitioning criteria, four mapping strategies and three variants of these strategies (thus a total of seven mapping strategies), we obtain the following 21 heuristics:

- Partc-Small, Partr-Small, Partw-Small,

- Partc-Snake-c, Partr-Snake-c, Partw-Snake-c, Partc-Snake-w, Partr-Snake-w, Partw-Snake-w, 


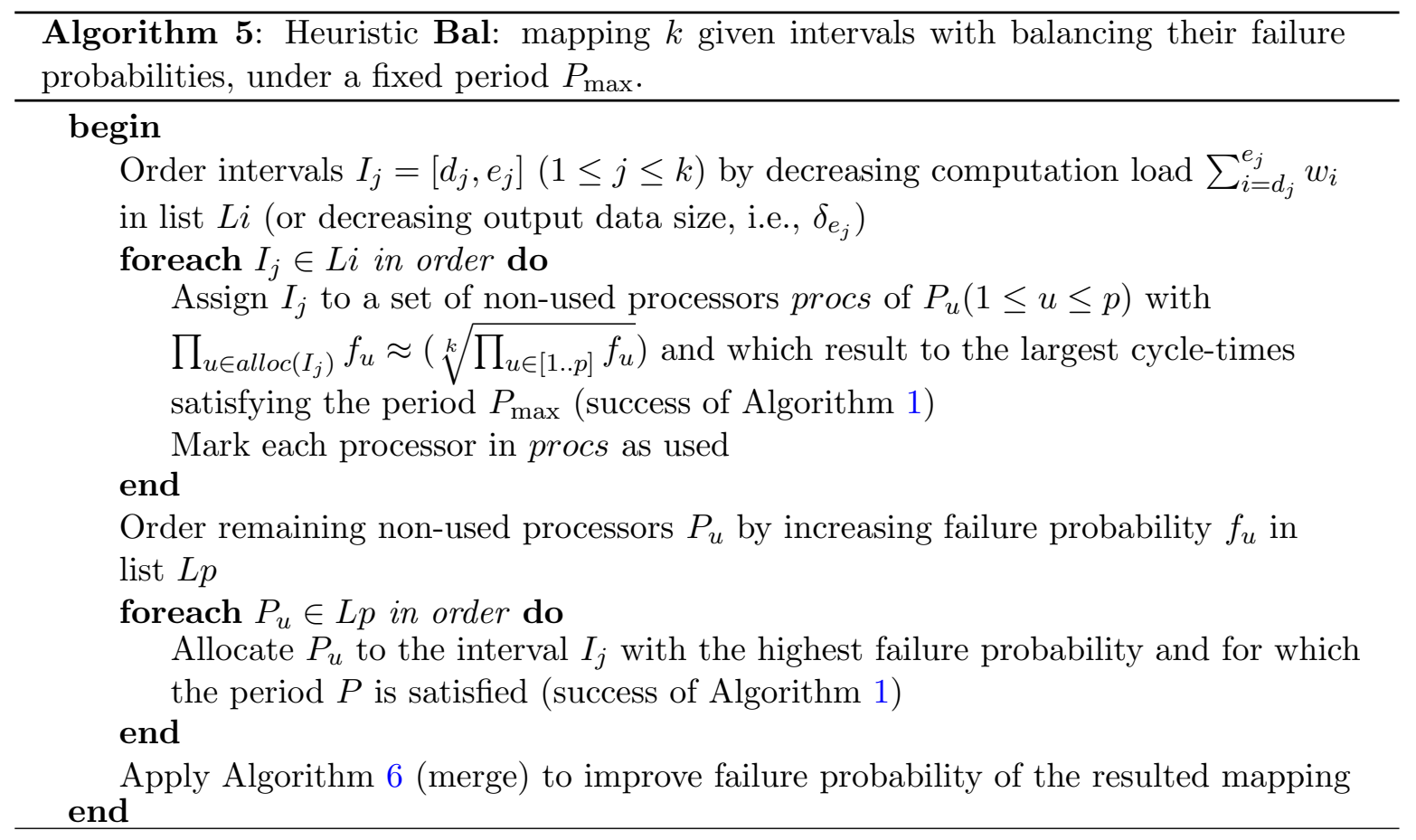

- Partc-BCT-c, Partr-BCT-c, Partw-BCT-c, Partc-BCT-w, Partr-BCT-w, Partw-BCT-w,

- Partc-Bal-c, Partr-Bal-c, Partw-Bal-c, Partc-Bal-w, Partr-Bal-w, Partw-Bal-w.

Algorithm 7 details the Partc-Small heuristic, and the others are working in a similar way, with different variants.

\subsection{Class 2: Heuristics with progressive creation of intervals}

In opposition to the previous heuristics, the heuristics of class 2 compute a mapping solution based on interleaved (and progressive) interval creations and processor allocations. Their principle is common. It consists in splitting stages within an interval into several intervals only if it is necessary, i.e., if no processor can be allocated without transgressing the period constraint. The objective is to minimize the number of intervals, so as to reach the smallest failure probability of a mapping.

In more details, each heuristic in the present class repeatedly attempts to assign an interval $I$ (initially composed of all stages $S_{1}, . ., S_{n}$ ) to an initial number $q$ of processors satisfying the period $P_{\max }$. If no processors are found, the heuristic splits interval $I$ into two new intervals, and try recursively to perform such an assignment. The process is repeated until processors are found for the interval, or no further split is possible. Because the final number of intervals is not known, $q$ may be chosen between 1 and $p$. To increase the probability to find processors to be allocated at each step, we have chosen $q$ between 1 and $\frac{p}{2}$. At the end, the heuristic attempts to assign the remaining non used processors (if any). It also tries to improve the failure probability of the resulted mapping. For that, it repeatedly merges the interval with the highest failure probability with previous or next intervals (Algorithm 6). 


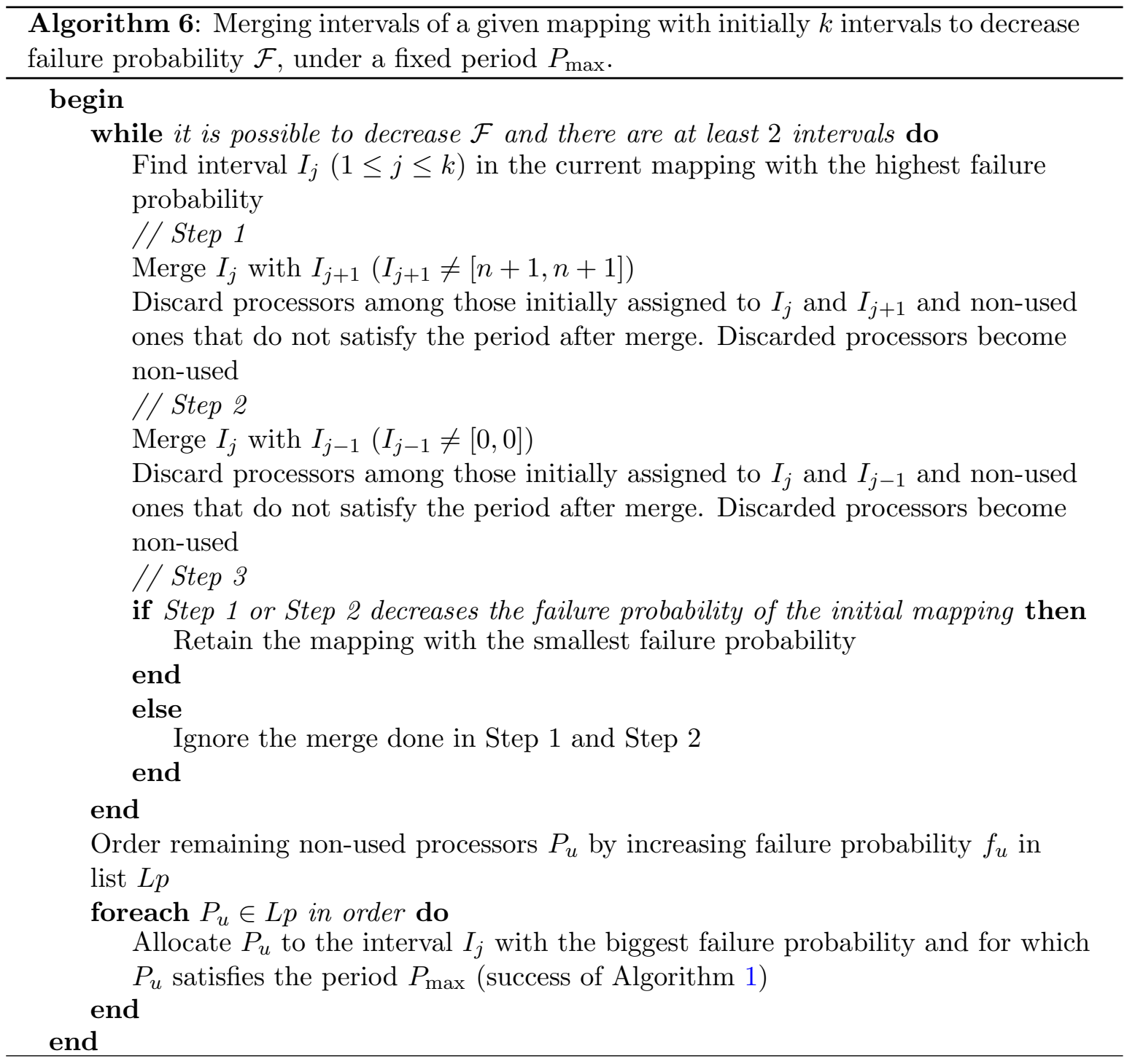




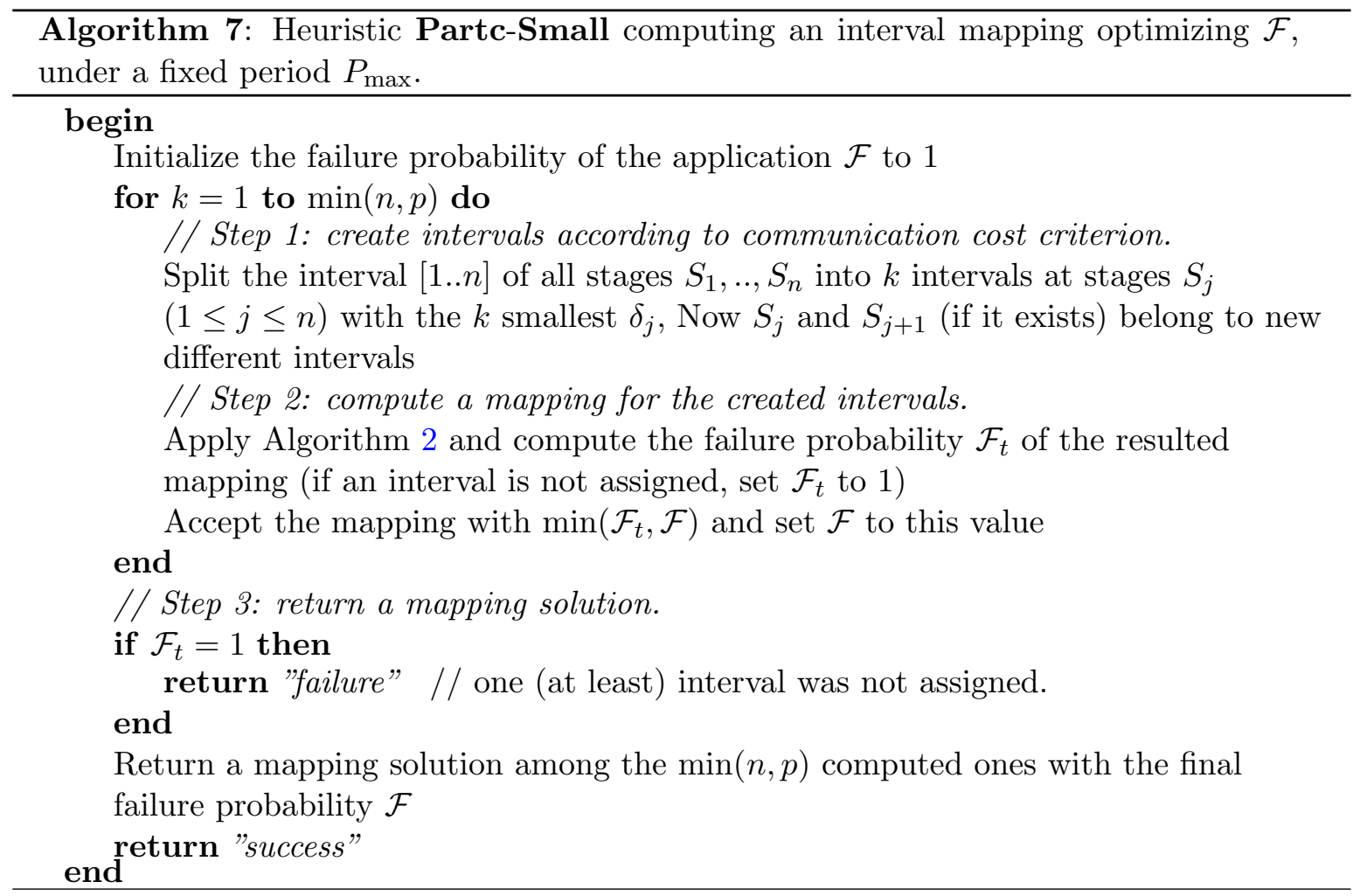

The merge process is done as long as the failure probability can be decreased and the period bound is still satisfied. Finally, the heuristic explores solutions for several values of $q$ (as explained above, $q$ varies from 1 to $\frac{p}{2}$ ) and it retains the solution with the smallest failure probability $\mathcal{F}$.

The way the split is done, as well as the mapping order of the step-by-step computed intervals, determine different heuristic variants in the present class. First, the split can be done at several places within an interval. In this work, we propose a recursive split of an interval into two intervals according to two criteria:

- Communication cost, where the split is done at the stage with the smallest output data size. The objective is to reduce costly communications. In the rest of this report, we identify heuristics using this split criteria by a prefix Splitc.

- Random partitioning, where the split is done at a randomly computed place. The objective is to have an intermediate solution between costly communications and costly computations. In the rest of this report, we identify heuristics using this split criteria by a prefix Splitr.

Secondly, we determine a mapping order of split intervals. In the present work, we defined two orders. The first order gives a mapping priority to the interval with the biggest output data size. Heuristics using this order are denoted with a suffix c. The second order gives the priority to the interval with the biggest workload $\left(\sum_{i=d_{j}}^{e_{j}} w_{i}\right)$. Heuristics using this order are denoted with a suffix $\mathbf{w}$. Therefore, the mapping priority is given to costly intervals in terms of either their output communications or their workload. Finally, we define the following four heuristics (including their variants): Splitc-c, Splitc-w, Splitr-c and Splitr-w. 


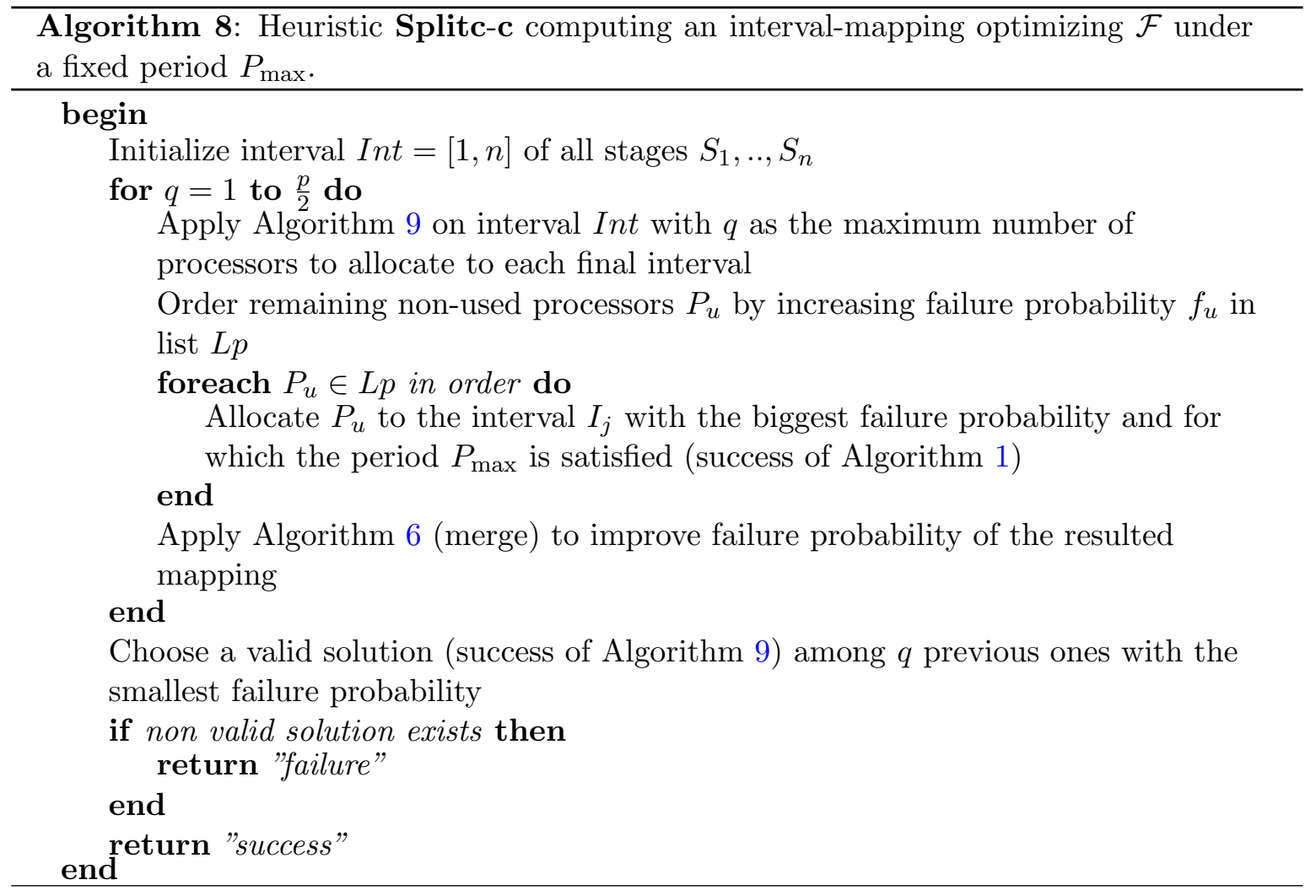

Heuristic Splitc-c is detailed in Algorithms 8 and 9. We recall that the heuristic explores solutions for different numbers of processors to be allocated per interval, and retains the solution with the smallest failure probability $\mathcal{F}$. 


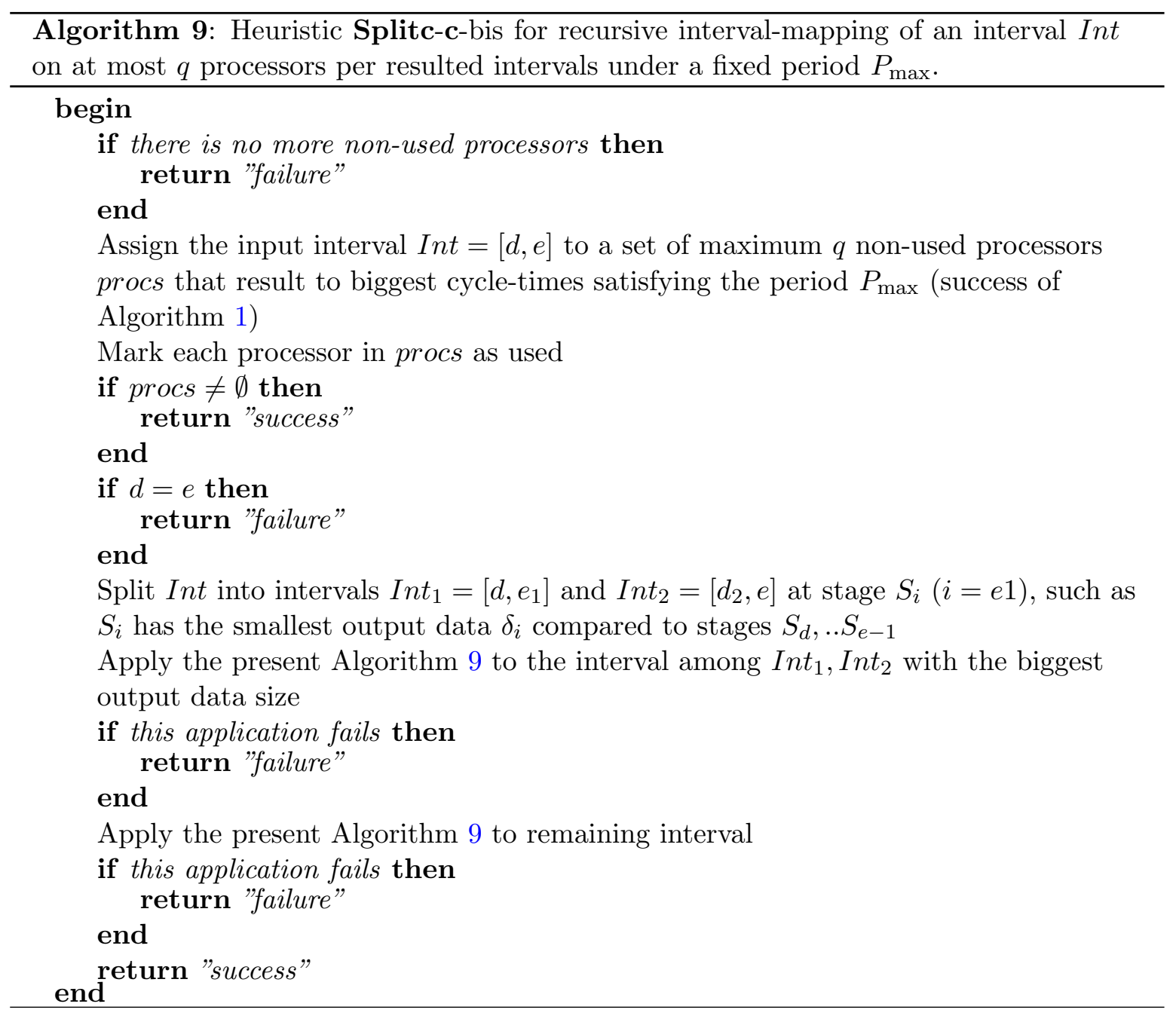




\section{Experiments}

This section discusses the performance of the heuristics proposed in Section 6 for different problems sizes. We have simulated several mapping scenarios for randomly generated applications with $n$ from 2 to 120 stages and randomly generated platforms with $p$ from 6 to 100 processors. For all these experiments, the computation load $(w)$ of stages is a random double chosen in the interval $[1,20]$ and the output data size $(\delta)$ or $d)$ is a random integer chosen in the interval $[1,25]$. As for computing resources, we recall that the heuristics have been designed for FullHet platforms. Experiments are done for such platforms, as well as for the restricted case of Failure Homogeneous ones. For each processor, the speed $(w)$ is a random double chosen in the interval $[1,20]$, and the input/output network card capacity is a random double chosen in the interval $[1,10]$, like the bandwidth $b$ of communication links. At last, the failure probability $(f)$ of processors are either homogeneous, equal to 0.1 , or a random value chosen between 0.05 and 0.3 .

Heuristics have been developed using the $\mathrm{C} / \mathrm{C}++$ language and gcc compiler version 4.3.2. Experiments have been conducted on two machines: one quad-processor machine (64bit AMD Opteron at 2.3GHz) with $32 \mathrm{~GB}$ of RAM and one quad-processor machine (64-bit AMD Opteron at $2.4 \mathrm{GHz}$ ) with $80 \mathrm{~GB}$ of RAM. The whole source code of the heuristics and the experiments setup can be found on the Web at: http://graal.ens-lyon.fr/ ${ }^{\text {hbouzian/ }}$ code/heuristics-FT-P.tgz.

In the following we first evaluate the performance of the heuristics compared to the optimal solution returned by the linear program presented in Section 5. Next, we focus on comparing the heuristics for large problem instances.

\subsection{Absolute performance of heuristics}

This section compares the results obtained by the heuristics to the optimal mapping solution returned by the linear program. This linear program is solved using the CPLEX Interactive Optimizer version 11.2.0. This version has a support for mixed integer linear programs like in the present case.

As the linear program has been designed for FailHom platforms (see Section 5), we limit the comparison with heuristics on such platforms. In addition, the large number of variables in the linear program forces us to limit the experiments to small applications and platforms. We have chosen scenarios with 8 stages and 10 processors. In particular, we selected 14 period bounds between 1.5 and 8.0. For each bound, 50 instances of application-platform pairs have been generated.

Table 1 reports the absolute deviation of the failure probabilities resulting from the heuristics compared to the optimal results. Several conclusions can be drawn. First, from the average (av.) and standard deviation (stdv.) columns, the heuristics based on the communication criteria to partition the stages into intervals (Partc-*) approach better the optimal solution. This is explained by the fact that small messages between intervals can increase the replication degree of each intervals and then the reliability of the mapping. Second, the best results are obtained by heuristics that repeatedly allocate processors one by one to all intervals. Indeed, the opposite behavior of the variants Partc-Bal- $\{\mathrm{c} \mid \mathrm{w}\}$ and those of class $2(\{$ Splitc $\mid$ Splitr $\}-\{\mathbf{c} \mid \mathbf{w}\})$ can more frequently limit the possibility to allocate processors. However, experiments presented later report that these heuristics can behave better for larger platforms. Therefore, the current experiment is not sufficient to determine the best heuristics 


\begin{tabular}{|l|c|c|c|c|c|c|}
\hline & \multicolumn{5}{|c|}{ Failure probability } & Execution time \\
\cline { 2 - 7 } & min & max & av. & stdv. & best rate & av. (msec) \\
\hline Partc-Small & 0.000 & 0.729 & 0.011 & 0.074 & $85.29 \%$ & 11.69 \\
\hline Partr-Small & 0.000 & 0.969 & 0.033 & 0.145 & $76.71 \%$ & 17.97 \\
\hline Partw-Small & 0.000 & 0.969 & 0.033 & 0.139 & $75.14 \%$ & 17.12 \\
\hline Partc-Snake-c & 0.000 & 0.729 & 0.007 & 0.050 & $84.14 \%$ & 3.36 \\
\hline Partc-Snake-w & 0.000 & 0.729 & 0.007 & 0.050 & $85.43 \%$ & 2.63 \\
\hline Partr-Snake-c & 0.000 & 0.900 & 0.030 & 0.132 & $75.14 \%$ & 4.66 \\
\hline Partr-Snake-w & 0.000 & 0.900 & 0.020 & 0.100 & $76.14 \%$ & 6.07 \\
\hline Partw-Snake-c & 0.000 & 0.969 & 0.032 & 0.137 & $73.14 \%$ & 2.56 \\
\hline Partw-Snake-w & 0.000 & 0.900 & 0.030 & 0.130 & $73.29 \%$ & 9.42 \\
\hline Partc-BCT-c & 0.000 & 0.891 & 0.010 & 0.066 & $83.86 \%$ & 4.42 \\
\hline Partc-BCT-w & 0.000 & 0.802 & 0.011 & 0.065 & $83.43 \%$ & 5.02 \\
\hline Partr-BCT-c & 0.000 & 0.900 & 0.025 & 0.116 & $74.43 \%$ & 3.12 \\
\hline Partr-BCT-w & 0.000 & 0.890 & 0.024 & 0.112 & $75.71 \%$ & 5.54 \\
\hline Partw-BCT-c & 0.000 & 0.969 & 0.030 & 0.130 & $71.86 \%$ & 8.66 \\
\hline Partw-BCT-w & 0.000 & 0.969 & 0.031 & 0.133 & $73.14 \%$ & 5.88 \\
\hline Partc-Bal-c & 0.000 & 0.900 & 0.016 & 0.096 & $82.43 \%$ & 8.64 \\
\hline Partc-Bal-w & 0.000 & 0.810 & 0.015 & 0.090 & $83.14 \%$ & 7.29 \\
\hline Partr-Bal-c & 0.000 & 0.891 & 0.027 & 0.123 & $73.71 \%$ & 6.57 \\
\hline Partr-Bal-w & 0.000 & 0.890 & 0.022 & 0.107 & $75.29 \%$ & 3.06 \\
\hline Partw-Bal-c & 0.000 & 0.900 & 0.034 & 0.136 & $70.00 \%$ & 8.29 \\
\hline Partw-Bal-w & 0.000 & 0.900 & 0.030 & 0.128 & $71.14 \%$ & 7.33 \\
\hline Splitc-c & 0.000 & 0.891 & 0.027 & 0.085 & $65.14 \%$ & 3.95 \\
\hline Splitc-w & 0.000 & 0.900 & 0.034 & 0.110 & $64.29 \%$ & 4.60 \\
\hline Splitr-c & 0.000 & 0.900 & 0.034 & 0.110 & $63.86 \%$ & 1.63 \\
\hline Splitr-w & 0.000 & 0.890 & 0.033 & 0.106 & $64.29 \%$ & 2.73 \\
\hline Linear-P & 0.000 & 0.000 & 0.000 & 0.000 & $100.00 \%$ & $2.78 \mathrm{e}+05$ \\
\hline
\end{tabular}

Table 1: Heuristics vs linear program results on a small Failure Homogeneous platform. Failure probabilities: minimum, maximum, average and standard absolute deviation for 700 mapping results. Execution times: average over the 700 executions.

in a general case. Third, from the success rates of heuristics to return the optimal mapping, we can conclude that most of the heuristics give satisfying results. At last, to confirm the above conclusions, Figure 9 shows the evolution of the average behavior of selected heuristics depending on the period bound. It can be noted that for period bounds higher than 2.5 , the behavior compared to the linear program varies. The main reason is that stage partitioning and processor distribution are done according to partially known data (communication and/or computation costs). Therefore, it is critical to identify one best heuristic for all period bounds.

Table 1 also reports the execution time of the heuristics and of the linear program. We see that the heuristics are very fast. The biggest average, $\approx 18 \mathrm{msec}$, is obtained when using the Small heuristic variants. This time is explained by the multiple attempts performed to randomly find a processor to be allocated to an interval. From the table, we also see that the linear program requires an average of some minutes $(\approx 5)$ to find the optimal solution. To explain this time, Figure 10 shows its evolution depending on the period bound. We can see that the average time considerably increases with the period bound. Indeed, when this bound is large, the solution space is wider, thus more operations are performed. The maximum resulted time is more than 3 hours, reached for a period bound set to 8 . This time is estimated to be very long for a small platform. This represents the main limitation of the linear program. For this reason, we limited experiments with the linear program to small problem instances. 


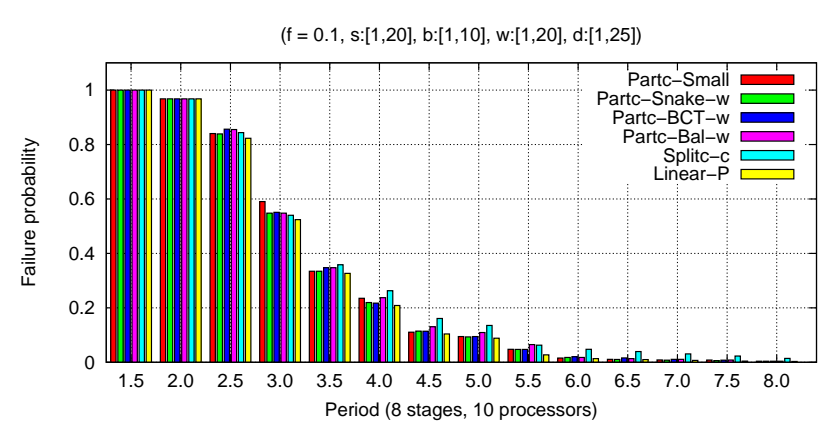

Figure 9: Best heuristics variants vs the linear program on small Failure Homogeneous platforms.

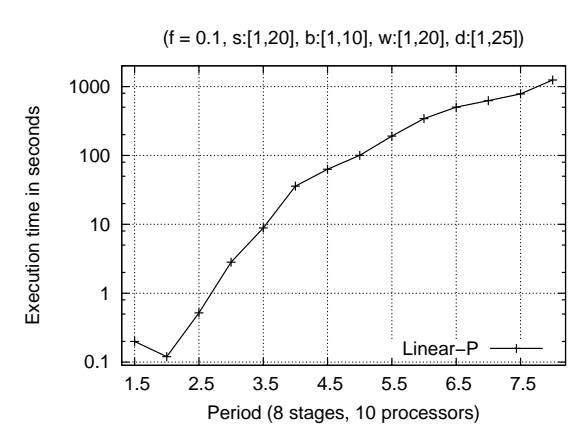

Figure 10: Execution times of the linear program.

\subsection{Comparisons between heuristics}

This section presents the results obtained for six sets of experiments. These sets correspond to scenarios with different platform and application sizes. We discuss the impact of varying these parameters on the performance of the heuristics. For each scenario, we also discuss the impact of the heterogeneity degree of processor failure probabilities. In this direction, each experiment set has been executed on both FullHet and FailHom platforms. All experiments have been executed on a same machine. In the following figures, each reported experimental value is an average of mapping failure probabilities over 200 application-platform pairs.

Figures 11, 12, 13, 14 and 15 compare the results obtained for the variants of each mapping heuristic presented in Section 6. There are six pairs of plots in each Figure. Each pair of plots corresponds to a set of experiments conduced on FailHom (on the left) and FullHet platforms (on the right). The first four pairs evaluate the performance of heuristics for fixed sizes of application-platform pairs and different period bounds, while the last two pairs evaluate the performance for fixed sizes of platforms (number of processors) and different applications sizes (number of stages) under a fixed period.

From each figure, we first observe that the behavior of heuristic variants is almost similar for both FailHom and FullHet platforms. This is explained by the fact that even if the failure probability of a processor is a relevant parameter considered by the mapping process, the respect of the period constraint has a more relevant impact on the result. Second, we observe that the average gap between the results of each heuristic variant becomes more important for medium and large platforms $(p \geq 50)$. That is mainly explained by the fact that when the number of processors increases, the order in which processors are allocated varies more, and the probability to allocate a same processor to a same interval decreases. Third, for the same platforms, we observe that heuristics Partc-* and Splitc-* globally reach better performance. These heuristics partition the stages (prefix Partc) or split the intervals (prefix Splitc) according to the communication criterion. In this case, the communication of large amount of data is avoided when possible, by mapping the two consecutive stages onto a same processor. For smaller communications, it is then possible to send more copies of the output data to the processor in charge of the next interval, while not exceeding the bound on the period. Therefore, it becomes easier to increase the replication degree of an interval, hence to reach a better reliability. Last, it is interesting to note that the random partitioning done by heuristics of class 1 (with a prefix Partr) can behave better than the heuristics that 


\begin{tabular}{|c|c|c|c|c|c|c|c|c|c|c|}
\hline & \multicolumn{5}{|c|}{ Small platforms (22800 results) } & \multicolumn{5}{|c|}{ Large platforms (14800 results) } \\
\hline & $\min$ & $\max$ & av. & stdv. & best rate & $\min$ & $\max$ & av. & stdv. & best rate \\
\hline Partc-Small & 0.000 & 0.969 & 0.076 & 0.184 & $59.79 \%$ & 0.000 & 0.958 & 0.065 & 0.100 & $14.77 \%$ \\
\hline Partr-Small & 0.000 & 0.990 & 0.093 & 0.191 & $4.84 \%$ & 0.000 & 0.862 & 0.114 & 0.123 & $0.75 \%$ \\
\hline Partw-Small & 0.000 & 0.988 & 0.093 & 0.194 & $3.20 \%$ & 0.000 & 0.995 & 0.215 & 0.271 & $0.71 \%$ \\
\hline Partc-Snake-c & 0.000 & 0.961 & 0.072 & 0.177 & $4.86 \%$ & 0.000 & 0.958 & 0.064 & 0.100 & $4.97 \%$ \\
\hline Partc-Snake-w & 0.000 & 0.951 & 0.057 & 0.154 & $3.97 \%$ & 0.000 & 0.958 & 0.045 & 0.087 & $10.45 \%$ \\
\hline Partr-Snake-c & 0.000 & 0.990 & 0.089 & 0.185 & $1.35 \%$ & 0.000 & 0.871 & 0.120 & 0.125 & $0.46 \%$ \\
\hline Partr-Snake-w & 0.000 & 0.990 & 0.071 & 0.159 & $1.75 \%$ & 0.000 & 0.886 & 0.099 & 0.112 & $0.88 \%$ \\
\hline Partw-Snake-c & 0.000 & 0.989 & 0.072 & 0.162 & $1.45 \%$ & 0.000 & 0.997 & 0.225 & 0.266 & $0.47 \%$ \\
\hline Partw-Snake-w & 0.000 & 0.988 & 0.064 & 0.151 & $1.41 \%$ & 0.000 & 0.995 & 0.209 & 0.262 & $0.50 \%$ \\
\hline Partc-BCT-c & 0.000 & 0.951 & 0.061 & 0.159 & $2.34 \%$ & 0.000 & 0.958 & 0.059 & 0.095 & $5.47 \%$ \\
\hline Partc-BCT-w & 0.000 & 0.951 & 0.056 & 0.153 & $2.10 \%$ & 0.000 & 0.958 & 0.052 & 0.090 & $8.38 \%$ \\
\hline Partr-BCT-c & 0.000 & 0.990 & 0.081 & 0.174 & $1.19 \%$ & 0.000 & 0.886 & 0.113 & 0.122 & $0.61 \%$ \\
\hline Partr-BCT-w & 0.000 & 0.900 & 0.069 & 0.155 & $1.17 \%$ & 0.000 & 0.862 & 0.102 & 0.111 & $0.52 \%$ \\
\hline Partw-BCT-c & 0.000 & 0.988 & 0.067 & 0.154 & $0.96 \%$ & 0.000 & 0.997 & 0.225 & 0.265 & $0.43 \%$ \\
\hline Partw-BCT-w & 0.000 & 0.988 & 0.061 & 0.146 & $0.88 \%$ & 0.000 & 0.997 & 0.221 & 0.261 & $0.42 \%$ \\
\hline Partc-Bal-c & 0.000 & 0.951 & 0.062 & 0.160 & $1.04 \%$ & 0.000 & 1.000 & 0.079 & 0.127 & $9.93 \%$ \\
\hline Partc-Bal-w & 0.000 & 0.951 & 0.058 & 0.154 & $1.44 \%$ & 0.000 & 0.958 & 0.075 & 0.118 & $7.34 \%$ \\
\hline Partr-Bal-c & 0.000 & 0.988 & 0.082 & 0.173 & $0.76 \%$ & 0.000 & 1.000 & 0.129 & 0.152 & $1.18 \%$ \\
\hline Partr-Bal-w & 0.000 & 0.988 & 0.070 & 0.156 & $0.92 \%$ & 0.000 & 1.000 & 0.118 & 0.134 & $0.75 \%$ \\
\hline Partw-Bal-c & 0.000 & 0.988 & 0.068 & 0.154 & $2.04 \%$ & 0.000 & 1.000 & 0.248 & 0.289 & $1.00 \%$ \\
\hline Partw-Bal-w & 0.000 & 0.990 & 0.062 & 0.146 & $0.44 \%$ & 0.000 & 1.000 & 0.240 & 0.285 & $0.56 \%$ \\
\hline Splitc-c & 0.000 & 0.931 & 0.050 & 0.102 & $2.34 \%$ & 0.000 & 0.777 & 0.074 & 0.088 & $11.48 \%$ \\
\hline Splitc-w & 0.000 & 0.909 & 0.052 & 0.106 & $1.43 \%$ & 0.000 & 0.882 & 0.053 & 0.076 & $17.64 \%$ \\
\hline Splitr-c & 0.000 & 0.985 & 0.076 & 0.143 & $0.95 \%$ & 0.000 & 0.871 & 0.181 & 0.155 & $0.27 \%$ \\
\hline Splitr-w & 0.000 & 0.990 & 0.074 & 0.139 & $1.23 \%$ & 0.000 & 0.871 & 0.177 & 0.151 & $0.28 \%$ \\
\hline
\end{tabular}

Table 2: Heuristics comparison over all done experiments (minimum, maximum, average and standard absolute deviation of failure probabilities for computed mappings).

partition stages according to computation costs criterion (with a prefix Partw). This often appears when the period bound is not too small (failure probability close to 1) nor too large (failure probability close to 0 ). This is explained by the fact that an important variation of stage workloads can easily lead to costly intervals when partitioning according to computation costs. Thus, the probability to find a processor matching the period bound may be reduced.

\subsection{Summary}

Table 2 sums up the performance of all heuristics over all experiments. For each heuristic, we have represented its absolute failure probability, and compared it to that of the heuristic reaching the best (smallest) probability for each experimental value. We have chosen this representation because it defines a meaningful lower bound for comparison. For small platforms, we observe that heuristic Partc-Small surpasses all the other heuristics in terms of success rate to give the smallest failure probability. However, from the average and standard deviation columns, other heuristics, like Splitc-c with a poor success rate, achieve better performance. The results are different for larger platforms. No large success rate is observed for one particular heuristic, but the rate is dispersed over multiple heuristics. However, we can deduce that heuristics partitioning stages or splitting intervals according to communication costs reach better performance. Among these heuristics, it is no obvious to distinguish a best one. Nevertheless, we observe satisfying results: with not-too-constrained periods, we reach failures probabilities less than 0.2 . 

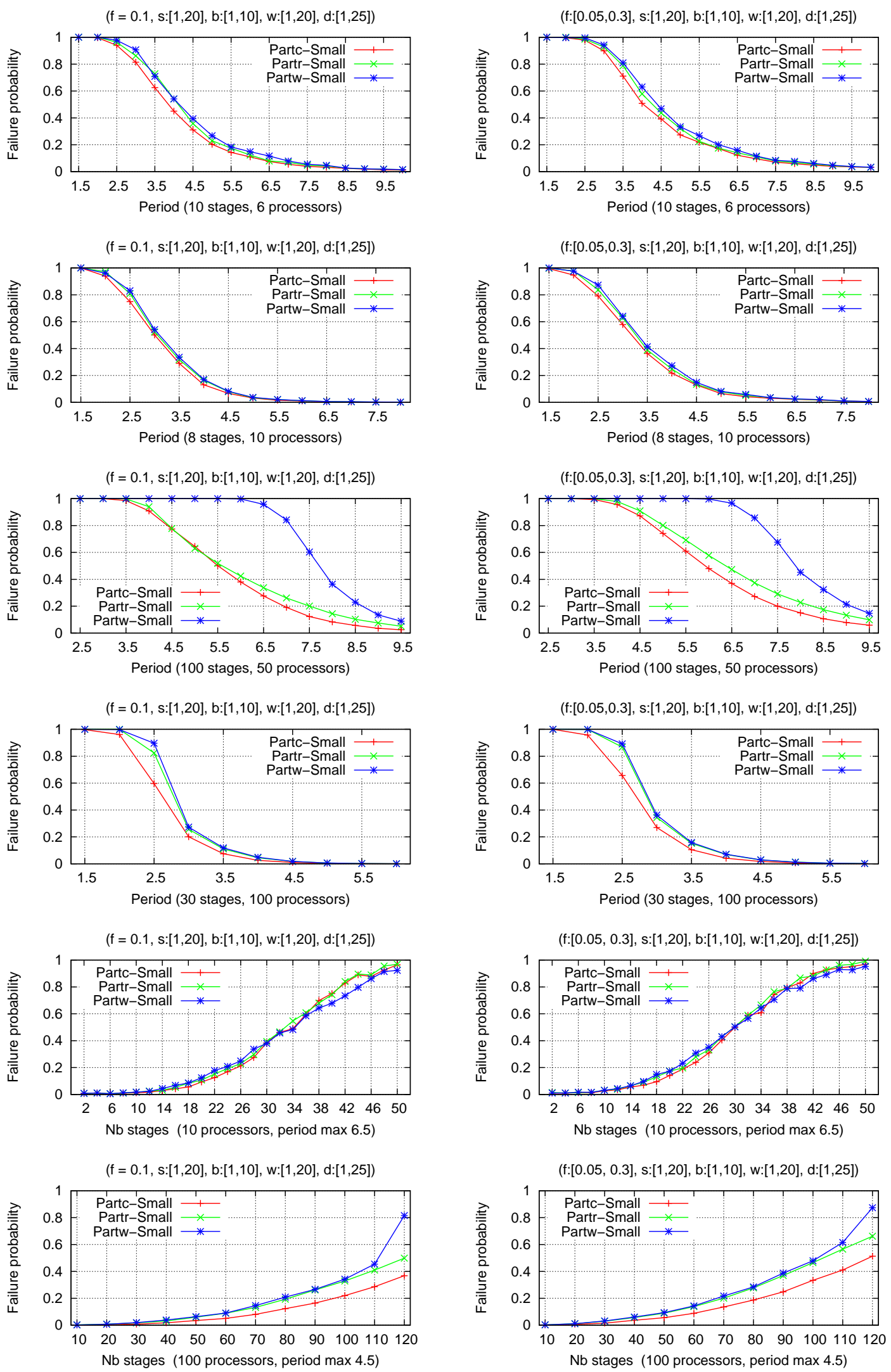

Figure 11: Comparison of $\{$ Partc|Partr|Partw $\}$-Small heuristic variants on Failure Homogeneous (right column) and FullHet (left column) platforms. 

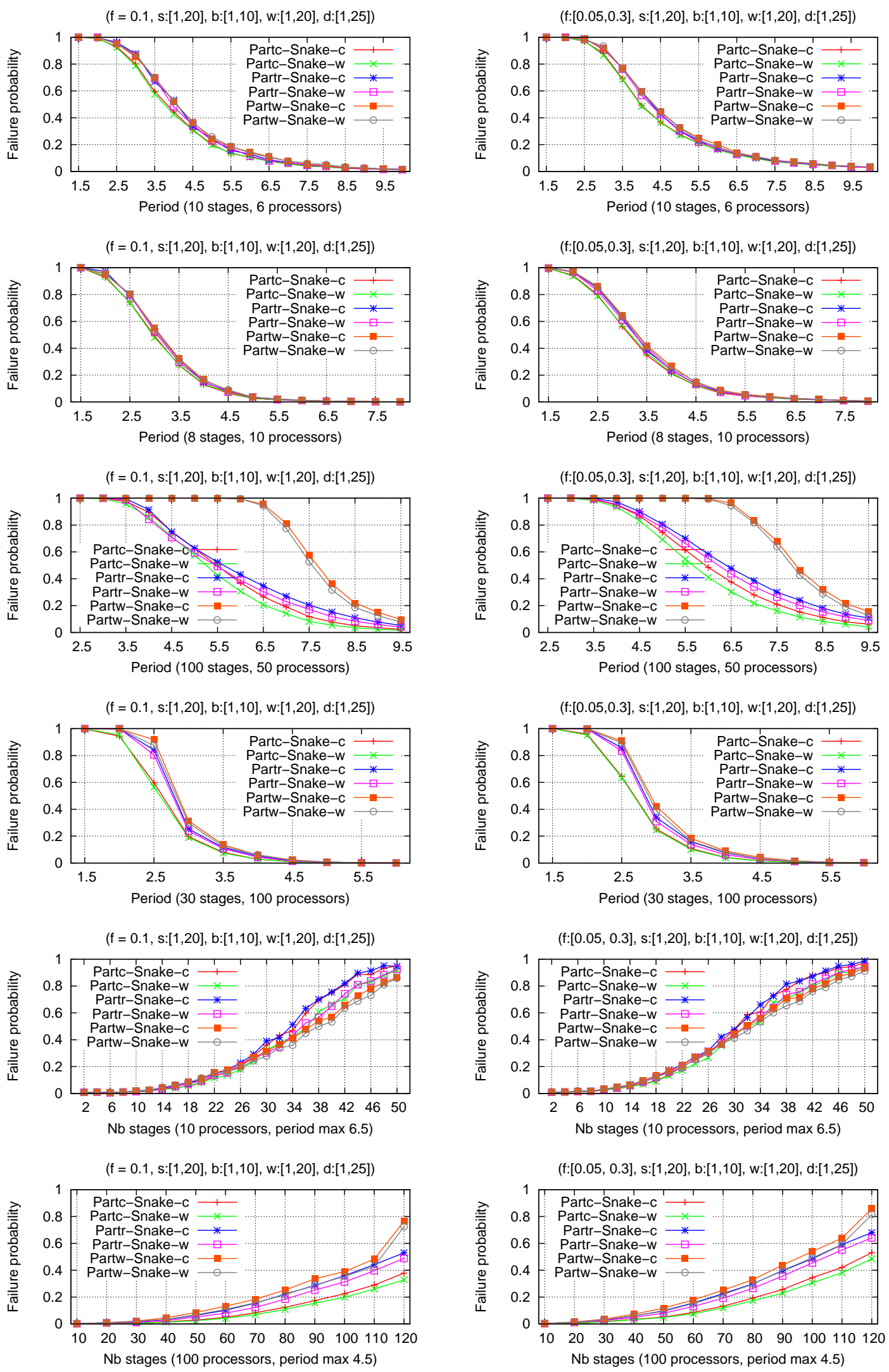

Figure 12: Comparison of $\{$ Partc $\mid$ Partr $\mid$ Partw $\}$-Snake- $\{\mathrm{c} \mid \mathrm{w}\}$ heuristic variants on Failure Homogeneous (right column) and FullHet (left column) platforms. 

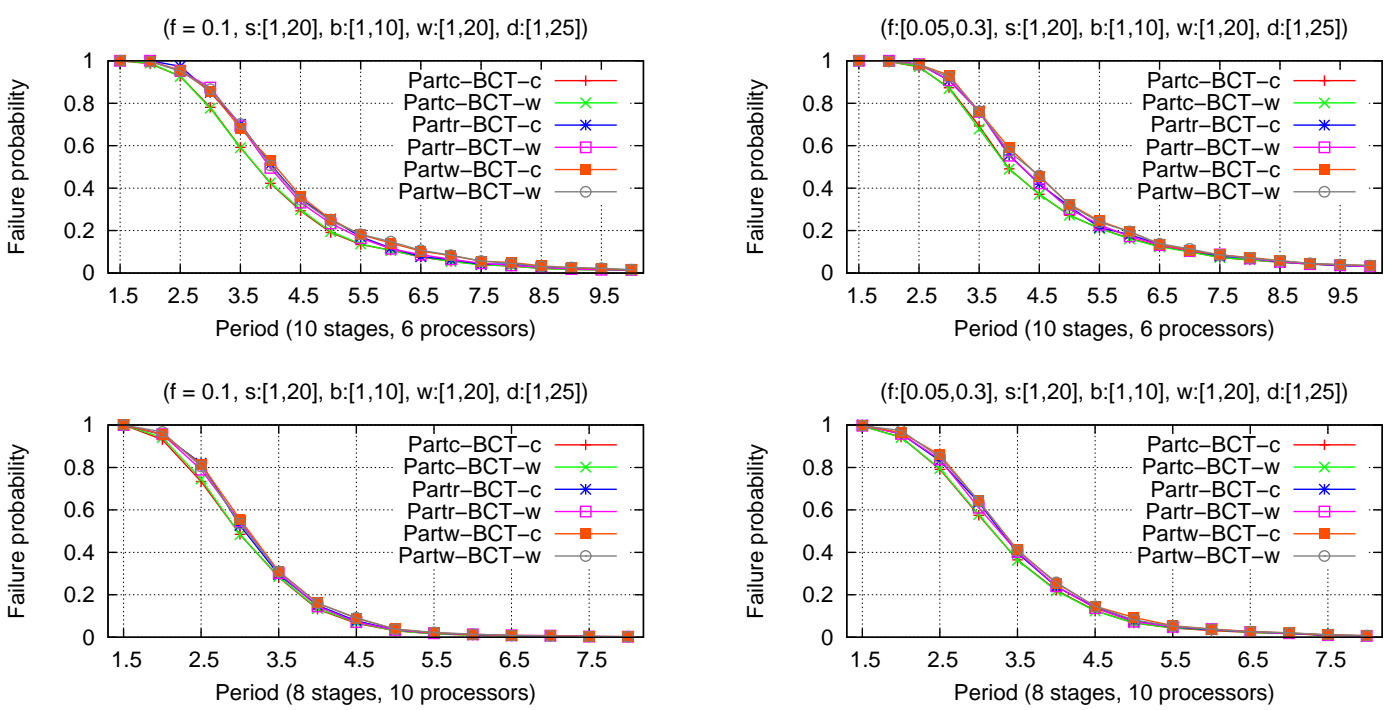

(f:[0.05,0.3], s:[1,20], b:[1,10], w:[1,20], d:[1,25])

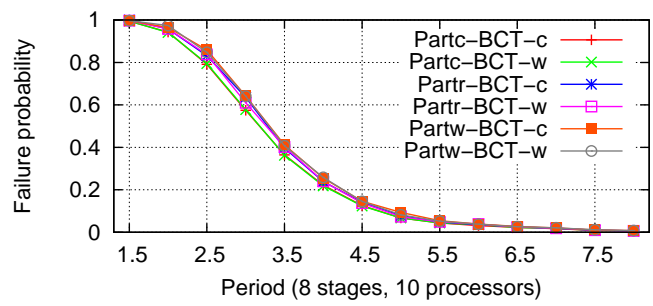

$(f=0.1, s:[1,20], b:[1,10], w:[1,20], d:[1,25])$

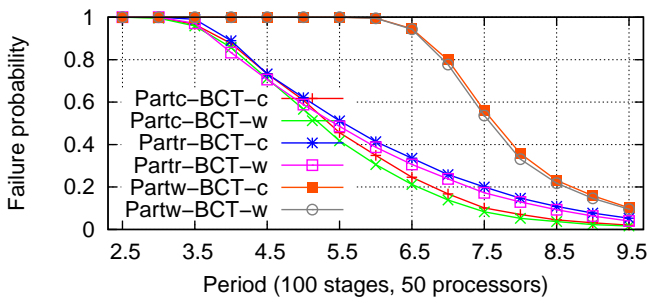

(f:[0.05,0.3], s:[1,20], b:[1,10], w:[1,20], d:[1,25])

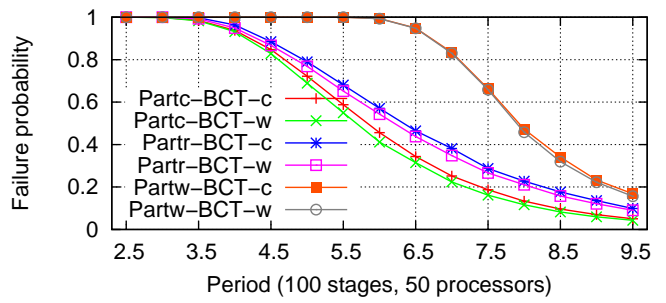

$(f=0.1, s:[1,20], b:[1,10], w:[1,20], d:[1,25])$

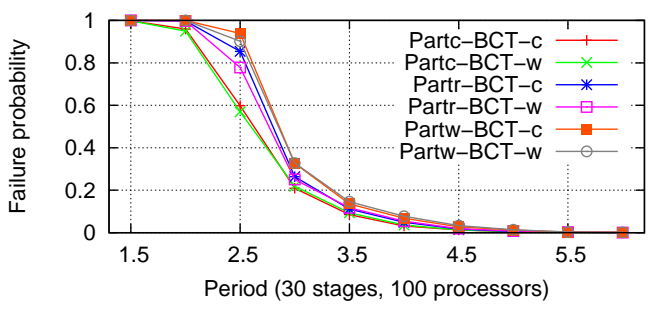

(f:[0.05,0.3], s:[1,20], b:[1,10], w:[1,20], d:[1,25])

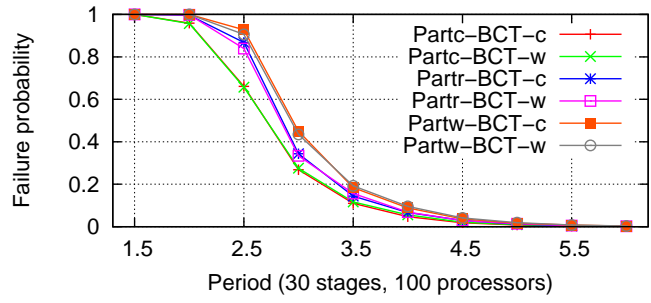

$(f=0.1, s:[1,20], b:[1,10], w:[1,20], d:[1,25])$

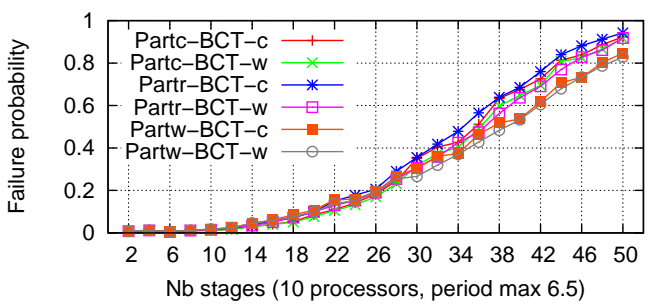

(f:[0.05, 0.3], s:[1,20], b:[1,10], w:[1,20], d:[1,25])

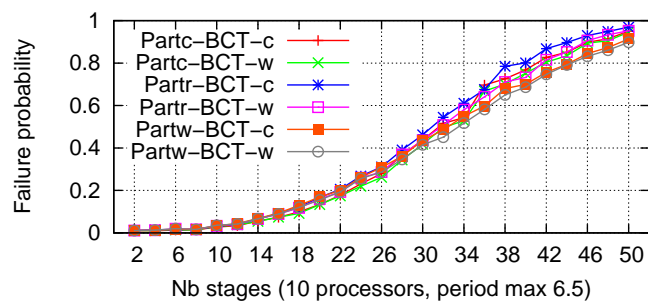

$(f=0.1, s:[1,20], b:[1,10], w:[1,20], d:[1,25])$

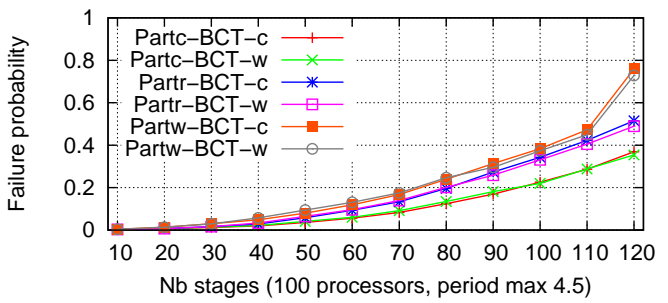

(f:[0.05, 0.3], s:[1,20], b:[1,10], w:[1,20], d:[1,25])

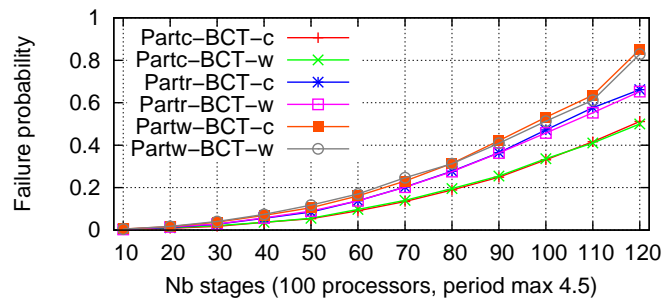

Figure 13: Comparison of $\{\operatorname{Partc}|\operatorname{Partr}| \mathbf{P a r t w}\}-\mathbf{B C T}-\{\mathrm{c} \mid \mathrm{w}\}$ heuristic variants on Failure Homogeneous (right column) and FullHet (left column) platforms. 

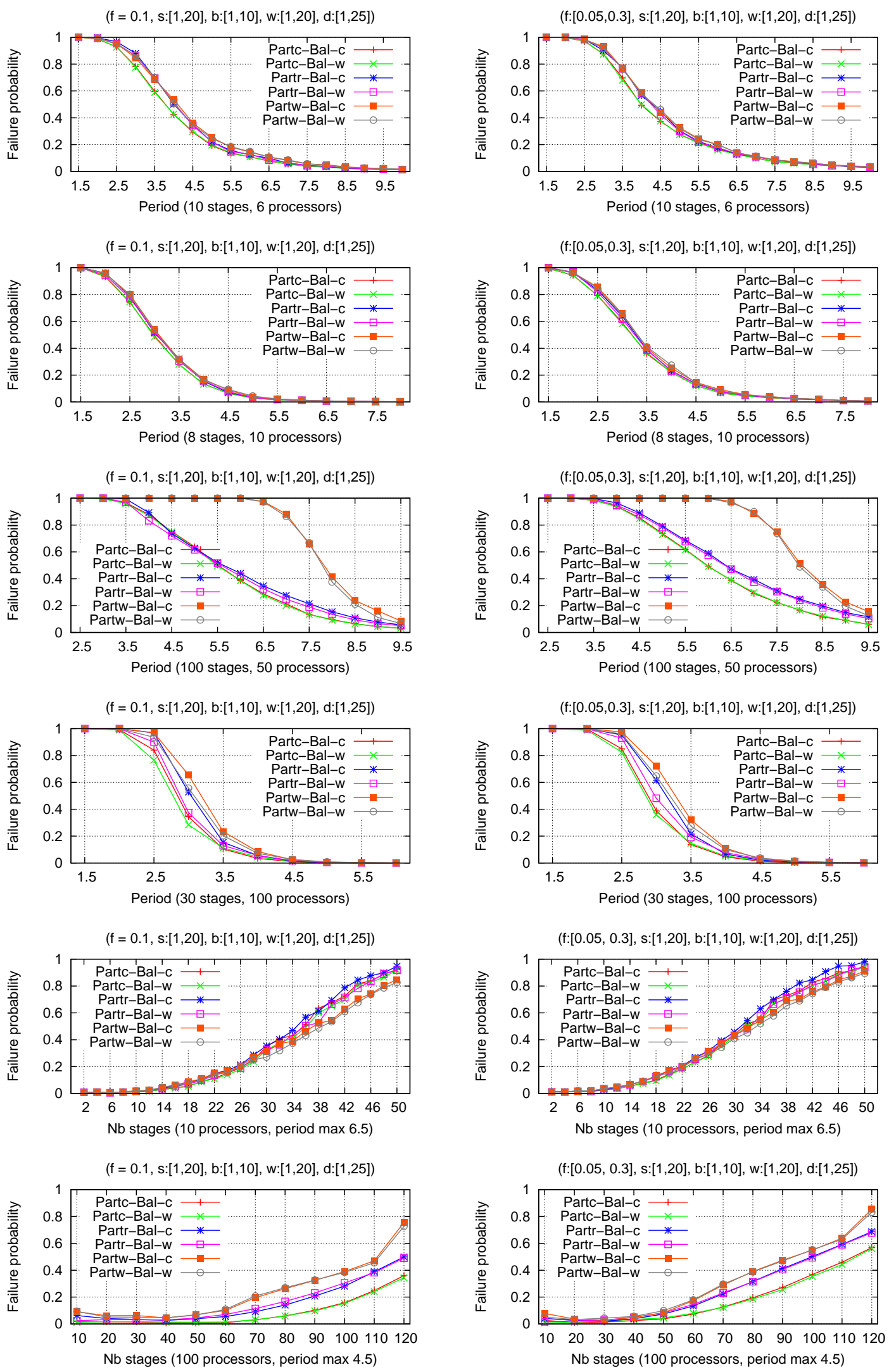

Figure 14: Comparison of $\{$ Partc $\mid$ Partr $\mid$ Partw $\}-B a l-\{c \mid w\}$ heuristic variants on Failure Homogeneous (right column) and FullHet (left column) platforms. 

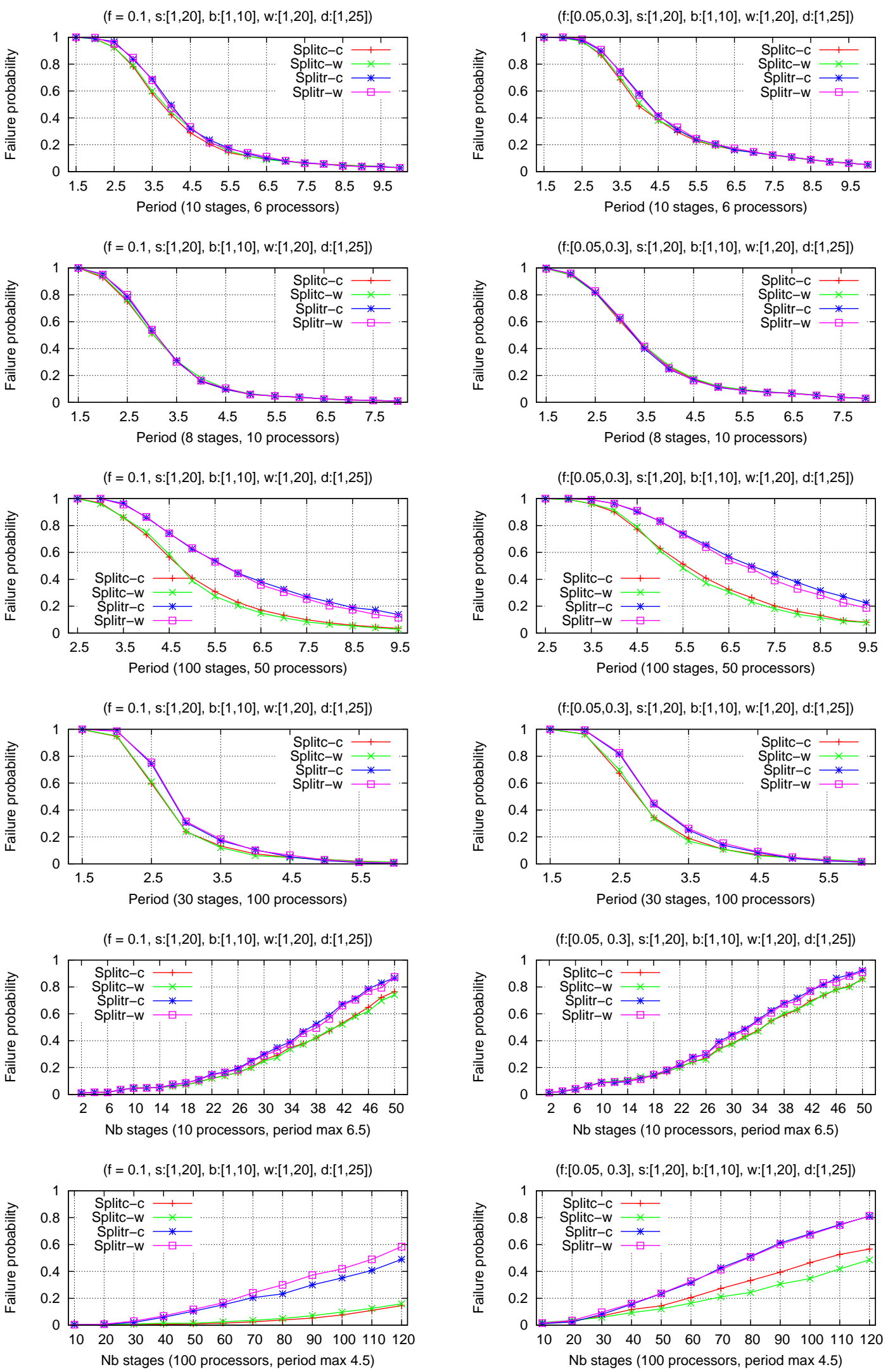

Figure 15: Comparison of $\{$ Splitc $\mid \operatorname{Splitr}\}-\{\mathbf{c} \mid \mathbf{w}\}$ heuristic variants on Failure Homogeneous (right column) and FullHet (left column) platforms. 


\section{Conclusion}

We have studied the complexity of the mapping problem onto heterogeneous platforms subject to failures. We focused on pipelined applications, composed of consecutive stages executed in a pipeline way. The objective is to find interval mapping solutions for such applications, while maximizing the reliability under a performance (throughput) constraint. A major difficulty is to deal with the impact of communication overheads. To the best of our knowledge, there are no previous results for this important bi-criteria problem, despite the fact that such pipeline workflows are widely encountered in real-life problems.

Our first contribution was to present new complexity results, providing a polynomial algorithm to solve the problem in a fully homogeneous setting, and proving the NP-hardness of the problem when adding one degree of heterogeneity. We also proposed a mixed integer linear programming formulation, which allows us to compute (in exponential time) the optimal solution on FailHom platforms. Even for such platforms, the program can take very long time to execute, even for small application/platform pairs, and we could not derive a formulation with a polynomial number of variables for FullHet platforms. At last, we have developed polynomial-time heuristics for fully heterogeneous platforms. Experimental results showed that for small FailHom platforms, the heuristics reach results close to the optimal solution provided by the linear program. Finally, we pointed out that for different problems sizes, multiple heuristics reach quite good results, and it is difficult to identify a particular one with "the best behavior" in all situations.

We are currently investigating an extension of the results to general mappings, where a processor may be assigned multiple intervals of stages. In this context, even computing the worst-case period of a given mapping becomes difficult, because it depends upon an exponential number of possible failure configurations. Therefore, much more work is needed before tackling the corresponding optimization problem: in a nutshell, before finding the best mapping, we have to agree on a polynomial approximation of the worst-case period of a given mapping! However, on the practical side, many of the heuristics presented in this report could be extended to this new problem. 


\section{References}

[1] B. Awerbuch, Y. Azar, A. Fiat, and F. Leighton. Making commitments in the face of uncertainty: how to pick a winner almost every time. In 28th ACM Symp. on Theory of Computing, pages 519-530. ACM Press, 1996.

[2] A. Benoit, L. Marchal, Y. Robert, and O. Sinnen. Mapping pipelined applications with replication to increase throughput and reliability. Research Report 2009-28, LIP, ENS Lyon, France, Oct. 2009. Available at graal.ens-lyon.fr/ abenoit.

[3] A. Benoit and Y. Robert. Mapping pipeline skeletons onto heterogeneous platforms. $J$. Parallel and Distributed Computing, 68(6):790-808, 2008.

[4] A. Benoit and Y. Robert. Complexity results for throughput and latency optimization of replicated and data-parallel workflows. Algorithmica, 2009. Available online at http://dx.doi.org/10.1007/s00453-008-9229-4.

[5] S. Bhatt, F. Chung, F. Leighton, and A. Rosenberg. On optimal strategies for cyclestealing in networks of workstations. IEEE Trans. Computers, 46(5):545-557, 1997.

[6] M. Cole. Bringing Skeletons out of the Closet: A Pragmatic Manifesto for Skeletal Parallel Programming. Parallel Computing, 30(3):389-406, 2004.

[7] M. R. Garey and D. S. Johnson. Computers and Intractability, a Guide to the Theory of NP-Completeness. W.H. Freeman and Company, 1979.

[8] B. Hong and V. Prasanna. Bandwidth-aware resource allocation for heterogeneous computing systems to maximize throughput. In Proceedings of the 32th International Conference on Parallel Processing (ICPP'2003). IEEE Computer Society Press, 2003.

[9] N. Karonis, B. Toonen, and I. Foster. MPICH-G2: A grid-enabled implementation of the message passing interface. J. Parallel and Distributed Computing, 63(5):551-563, 2003.

[10] F. Rabhi and S. Gorlatch. Patterns and Skeletons for Parallel and Distributed Computing. Springer Verlag, 2002.

[11] A. Rosenberg. Optimal schedules for cycle-stealing in a network of workstations with a bag-of-tasks workload. IEEE Trans. Parallel and Distributed Systems, 13(2):179-191, 2002 .

[12] J. Subhlok and G. Vondran. Optimal mapping of sequences of data parallel tasks. In Proc. 5th ACM SIGPLAN Symposium on Principles and Practice of Parallel Programming, 1995.

[13] J. Subhlok and G. Vondran. Optimal latency-throughput tradeoffs for data parallel pipelines. In ACM Symposium on Parallel Algorithms and Architectures, 1996.

[14] G. Tel. Introduction to Distributed Algorithms. Cambridge University Press, 2000. 